\title{
On Transience of Lévy-Type Processes
}

\author{
Nikola Sandrić \\ Institut für Mathematische Stochastik \\ Fachrichtung Mathematik, Technische Universität Dresden, 01062 Dresden, Germany \\ and \\ Department of Mathematics \\ Faculty of Civil Engineering, University of Zagreb, 10000 Zagreb, Croatia \\ Email: nsandric@grad.hr
}

April 14, 2016

\begin{abstract}
In this paper, we study weak and strong transience of a class of Feller processes associated with pseudo-differential operators, the so-called Lévy-type processes. As a main result, we derive Chung-Fuchs type conditions (in terms of the symbol of the corresponding pseudo-differential operator) for these properties, which are sharp for Lévy processes. Also, as a consequence, we discuss the weak and strong transience with respect to the dimension of the state space and Pruitt indices, thus generalizing some well-known results related to elliptic diffusion and stable Lévy processes. Finally, in the case when the symbol is radial (in the co-variable) we provide conditions for the weak and strong transience in terms of the Lévy measures.
\end{abstract}

AMS 2010 Mathematics Subject Classification: 60J25, 60J75, 60G17

Keywords and phrases: Lévy-type process, strong transience, symbol, transience, weak transience

\section{Introduction}

Let $\left(\left\{L_{t}\right\}_{t \geq 0}, \mathbb{P}\right)$ be a $d$-dimensional Lévy process. The process $\left\{L_{t}\right\}_{t \geq 0}$ is said to be transient if

$$
\mathbb{P}\left(\lim _{t \longrightarrow \infty}\left|L_{t}\right|=\infty\right)=1,
$$

and recurrent if

$$
\mathbb{P}\left(\liminf _{t \longrightarrow \infty}\left|L_{t}\right|=0\right)=1 .
$$

It is well known that every Lévy process is either transient or recurrent (see [Sat99, Theorem 35.3]). The transience and recurrence of Lévy processes can also be (equivalently) characterized through last exit times. Let $O \subseteq \mathbb{R}^{d}$ be arbitrary. Define $L_{O}:=\sup \left\{t \geq 0: L_{t} \in O\right\}$, the last exit time of $\left\{L_{t}\right\}_{t \geq 0}$ from the set $O$. Clearly, if $O$ is open, then, since $\left\{L_{t}\right\}_{t \geq 0}$ has càdlàg sample paths, $L_{O}$ becomes a random variable. Now, we easily see that $\left\{L_{t}\right\}_{t \geq 0}$ is transient if, and only if, $L_{B(0, r)}<\infty \mathbb{P}$-a.s. for every $r>0$. Here, $B(x, r)$ denotes the open ball around $x \in \mathbb{R}^{d}$ of radius $r>0$. The previous observation suggests that the last exit times might be suitable objects for further and deeper analysis of the transience property. Indeed, in [SW04] it has been shown that 
for a (transient) Lévy process $\left\{L_{t}\right\}_{t \geq 0}$ and any $\kappa>0, \mathbb{E}\left[L_{B(0, r)}^{\kappa}\right]$ is either finite or infinite for every $r>0$ (see also [Haw77] for the case of symmetric Lévy processes). Accordingly, a (transient) Lévy process $\left\{L_{t}\right\}_{t \geq 0}$ is said to be $\kappa$-weakly transient if

$$
\mathbb{E}\left[L_{B(0, r)}^{\kappa}\right]=\infty \text { for all } r>0,
$$

and $\kappa$-strongly transient if

$$
\mathbb{E}\left[L_{B(0, r)}^{\kappa}\right]<\infty \text { for all } r>0 .
$$

Also, in the same reference, the authors have proved that the above conditions are equivalent to

$$
\int_{0}^{\infty} t^{\kappa} \mathbb{P}\left(L_{t} \in B(0, r)\right) d t=\infty \quad \text { for all } r>0
$$

and

$$
\int_{0}^{\infty} t^{\kappa} \mathbb{P}\left(L_{t} \in B(0, r)\right) d t<\infty \quad \text { for all } r>0,
$$

respectively. However, the above characterizations are in general not too operable, that is, it is practically very hard to say (based just on the above characterizations) whether a given Lévy process is $\kappa$-weakly or $\kappa$-strongly transient. Therefore, they also derive certain analytic conditions for theses properties: (i) Chung-Fuchs type conditions (in terms of the corresponding characteristic exponent) and, in the one-dimensional symmetric case, (ii) conditions in terms of the underlying Lévy measure. Let us also remark that in their follow up paper [SW05] they have made a deep analysis of the $\kappa$-weak and $\kappa$-strong transience of (semi-)stable Lévy processes (see also [Tak67] for the case of rotationally invariant stable Lévy processes). The main goal of this paper is to extend the notion of the $\kappa$-weak and $\kappa$-strong transience, and derive certain more operable (analytic) conditions for these properties (which generalize the conditions presented in [SW04]), to a class of Feller processes, the so-called Lévy-type processes.

A $d$-dimensional Feller process is a strong Markov process $\left(\left\{F_{t}\right\}_{t \geq 0},\left\{\mathbb{P}^{x}\right\}_{x \in \mathbb{R}^{d}}\right)$ with state space $\left(\mathbb{R}^{d}, \mathcal{B}\left(\mathbb{R}^{d}\right)\right)$ whose associated operator semigroup $\left\{P_{t}\right\}_{t \geq 0}$,

$$
P_{t} f(x):=\mathbb{E}^{x}\left[f\left(F_{t}\right)\right], \quad t \geq 0, x \in \mathbb{R}^{d}, f \in C_{\infty}\left(\mathbb{R}^{d}\right),
$$

forms a Feller semigroup. This means that

(i) $\left\{P_{t}\right\}_{t \geq 0}$ enjoys the Feller property, that is, $P_{t}\left(C_{\infty}\left(\mathbb{R}^{d}\right)\right) \subseteq C_{\infty}\left(\mathbb{R}^{d}\right)$ for all $t \geq 0$;

(ii) $\left\{P_{t}\right\}_{t \geq 0}$ is strongly continuous, that is, $\lim _{t \rightarrow 0}\left\|P_{t} f-f\right\|_{\infty}=0$ for all $f \in C_{\infty}\left(\mathbb{R}^{d}\right)$.

Here, $\mathcal{B}\left(\mathbb{R}^{d}\right), C_{\infty}\left(\mathbb{R}^{d}\right)$ and $\|\cdot\|_{\infty}$ denote the Borel $\sigma$-algebra on $\mathbb{R}^{d}$, space of continuous functions vanishing at infinity and supremum norm, respectively. Note that every Feller semigroup $\left\{P_{t}\right\}_{t>0}$ can be uniquely extended to the space of bounded and Borel measurable functions $B_{b}\left(\mathbb{R}^{d}\right.$ ) (see [Sch98a, Section 3]). For notational simplicity, we denote this extension again by $\left\{P_{t}\right\}_{t \geq 0}$. Also, let us remark that every Feller process has càdlàg sample paths (see [Jac05, Theorems 3.4.19]). The infinitesimal generator $\left(\mathcal{A}, \mathcal{D}_{\mathcal{A}}\right)$ of a Feller process $\left\{F_{t}\right\}_{t \geq 0}$ (or of the corresponding Feller semigroup $\left.\left\{P_{t}\right\}_{t \geq 0}\right)$ is a linear operator $\mathcal{A}: \mathcal{D}_{\mathcal{A}} \longrightarrow C_{\infty}\left(\mathbb{R}^{d}\right)$ defined by

$$
\mathcal{A} f:=\lim _{t \longrightarrow 0} \frac{P_{t} f-f}{t}, \quad f \in \mathcal{D}_{\mathcal{A}}:=\left\{f \in C_{\infty}\left(\mathbb{R}^{d}\right): \lim _{t \longrightarrow 0} \frac{P_{t} f-f}{t} \text { exists in }\|\cdot\|_{\infty}\right\} .
$$

Let $C_{c}^{\infty}\left(\mathbb{R}^{d}\right)$ be the space of smooth functions with compact support. Under the assumption 
(C1) $C_{c}^{\infty}\left(\mathbb{R}^{d}\right) \subseteq \mathcal{D}_{\mathcal{A}}$,

in [Cou66, Theorem 3.4] it has been shown that $\left.\mathcal{A}\right|_{C_{c}^{\infty}\left(\mathbb{R}^{d}\right)}$ is a pseudo-differential operator, that is, it can be written in the form

$$
\left.\mathcal{A}\right|_{C_{C}^{\infty}\left(\mathbb{R}^{d}\right)} f(x)=-\int_{\mathbb{R}^{d}} q(x, \xi) e^{i\langle\xi, x\rangle} \mathcal{F}(f)(\xi) d \xi,
$$

where $\mathcal{F}(f)(\xi):=(2 \pi)^{-d} \int_{\mathbb{R}^{d}} e^{-i\langle\xi, x\rangle} f(x) d x$ denotes the Fourier transform of the function $f(x)$. The function $q: \mathbb{R}^{d} \times \mathbb{R}^{d} \longrightarrow \mathbb{C}$ is called the symbol of the pseudo-differential operator. It is measurable and locally bounded in $(x, \xi)$ and continuous and negative definite as a function of $\xi$. Hence, by [Jac01, Theorem 3.7.7], the function $\xi \longmapsto q(x, \xi)$ has for each $x \in \mathbb{R}^{d}$ the following Lévy-Khintchine representation

$$
q(x, \xi)=a(x)-i\langle\xi, b(x)\rangle+\frac{1}{2}\langle\xi, C(x) \xi\rangle-\int_{\mathbb{R}^{d}}\left(e^{i\langle\xi, y\rangle}-1-i\langle\xi, y\rangle 1_{B(0,1)}(y)\right) \nu(x, d y),
$$

where $a(x)$ is a nonnegative Borel measurable function, $b(x)$ is an $\mathbb{R}^{d}$-valued Borel measurable function, $C(x):=\left(c_{i j}(x)\right)_{1 \leq i, j \leq d}$ is a symmetric non-negative definite $d \times d$ matrix-valued Borel measurable function and $\nu(x, d y)$ is a Borel kernel on $\mathbb{R}^{d} \times \mathcal{B}\left(\mathbb{R}^{d}\right)$, called the Lévy measure, satisfying

$$
\nu(x,\{0\})=0 \quad \text { and } \quad \int_{\mathbb{R}^{d}} \min \left\{1,|y|^{2}\right\} \nu(x, d y)<\infty, \quad x \in \mathbb{R}^{d} .
$$

The quadruple $(a(x), b(x), C(x), \nu(x, d y))$ is called the Lévy quadruple of the pseudo-differential operator $\left.\mathcal{A}\right|_{C_{c}^{\infty}\left(\mathbb{R}^{d}\right)}$ (or of the symbol $q(x, \xi)$ ). In the sequel we also assume the following two conditions on the symbol $q(x, \xi)$ :

(C2) $\|q(\cdot, \xi)\|_{\infty} \leq c\left(1+|\xi|^{2}\right)$ for all $\xi \in \mathbb{R}^{d}$ and some $c \geq 0$;

(C3) $q(x, 0)=0$ for all $x \in \mathbb{R}^{d}$.

The condition in (C2) means that $q(x, \xi)$ has bounded coefficients (see [Sch98b, Lemma 2.1]), while, according to [Sch98a, Theorem 5.2], (C3) (together with $(\mathbf{C 2})$ ) implies that $\left\{F_{t}\right\}_{t \geq 0}$ is conservative, that is, $\mathbb{P}^{x}\left(F_{t} \in \mathbb{R}^{d}\right)=1$ for all $t \geq 0$ and all $x \in \mathbb{R}^{d}$. Note that the conservativeness is a natural assumption in the context of problems we are concerned in this paper. Further, observe that in the case when the symbol $q(x, \xi)$ does not depend on the variable $x \in \mathbb{R}^{d},\left\{F_{t}\right\}_{t>0}$ becomes a Lévy process. Moreover, every Lévy process is uniquely and completely characterized through its corresponding symbol (see [Sat99, Theorems 7.10 and 8.1]). According to this, it is not hard to check that every Lévy process satisfies condition (C1) (see [Sat99, Theorem 31.5]). Thus, the class of processes we consider in this paper contains a subclass of Lévy processes. Let us also remark here that, unlike in the case of Lévy processes, it is not possible to associate a Feller process to every symbol (see for [BSW13] details). Throughout this paper, the symbol $\left\{F_{t}\right\}_{t \geq 0}$ denotes a Feller process satisfying conditions $(\mathbf{C 1}),(\mathbf{C 2})$ and $(\mathbf{C 3})$. Such a process is called a Lévy-type process. Also, a Lévy process is denoted by $\left\{L_{t}\right\}_{t \geq 0}$. If $\nu(x, d y)=0$ for all $x \in \mathbb{R}^{d}$, according to [BSW13, Theorem 2.44], $\left\{F_{t}\right\}_{t \geq 0}$ becomes an elliptic diffusion process. Note that this definition agrees with the standard definition of elliptic diffusion processes (see [RW00a]). For more on Lévytype processes we refer the readers to the monograph [BSW13].

Now, let us recall the definitions of transience and recurrence of Markov processes. Let $\left(\left\{M_{t}\right\}_{t \geq 0}\right.$, $\left.\left\{\mathbb{P}^{x}\right\}_{x \in \mathbb{R}^{d}}\right)$ be a Markov process with càdlàg sample paths on the state space $\left(\mathbb{R}^{d}, \mathcal{B}\left(\mathbb{R}^{d}\right)\right)$, where $d \geq 1$. The process $\left\{M_{t}\right\}_{t \geq 0}$ is called 
(i) irreducible if there exists a $\sigma$-finite measure $\varphi(d y)$ on $\mathcal{B}\left(\mathbb{R}^{d}\right)$ such that whenever $\varphi(B)>0$ we have $\int_{0}^{\infty} \mathbb{P}^{x}\left(M_{t} \in B\right) d t>0$ for all $x \in \mathbb{R}^{d}$.

(ii) transient if it is $\varphi$-irreducible and if there exists a countable covering of $\mathbb{R}^{d}$ with sets $\left\{B_{j}\right\}_{j \in \mathbb{N}} \subseteq$ $\mathcal{B}\left(\mathbb{R}^{d}\right)$, such that for each $j \in \mathbb{N}$ there is a finite constant $c_{j} \geq 0$ such that $\int_{0}^{\infty} \mathbb{P}^{x}\left(M_{t} \in B_{j}\right) d t \leq$ $c_{j}$ holds for all $x \in \mathbb{R}^{d}$.

(iii) recurrent if it is $\varphi$-irreducible and if $\varphi(B)>0$ implies $\int_{0}^{\infty} \mathbb{P}^{x}\left(M_{t} \in B\right) d t=\infty$ for all $x \in \mathbb{R}^{d}$.

It is well known that every irreducible Markov process is either transient or recurrent (see [Twe94, Theorem 2.3]). Also, let us remark that if $\left\{M_{t}\right\}_{t \geq 0}$ is a $\varphi$-irreducible Markov process, then the irreducibility measure $\varphi(d y)$ can be maximized, that is, there exists a unique "maximal" irreducibility measure $\psi(d y)$ such that for any measure $\bar{\varphi}(d y),\left\{M_{t}\right\}_{t \geq 0}$ is $\bar{\varphi}$-irreducible if, and only if, $\bar{\varphi} \ll \psi$ (see [Twe94, Theorem 2.1]). According to this, from now on, when we refer to irreducibility measure we actually refer to the maximal irreducibility measure. In the sequel, we consider only the so-called open-set irreducible Markov processes, that is, Markov processes whose maximal irreducibility measure is fully supported. An example of such measure is the Lebesgue measure, which we denote by $\lambda(d y)$. Clearly, a Markov process $\left\{M_{t}\right\}_{t \geq 0}$ will be $\lambda$-irreducible if $\mathbb{P}^{x}\left(M_{t} \in B\right)>0$ for all $t>0$ and all $x \in \mathbb{R}^{d}$ whenever $\lambda(B)>0$. In particular, the process $\left\{M_{t}\right\}_{t \geq 0}$ will be $\lambda$-irreducible if the transition kernel $\mathbb{P}^{x}\left(M_{t} \in d y\right), t>0, x \in \mathbb{R}^{d}$, possesses a strictly positive transition density function. Let us remark here that the $\lambda$-irreducibility of Lévy-type processes is a very well-studied topic in the literature. We refer the readers to [She91] and [ST97] for the case of elliptic diffusion processes, to [Kol00] for the case of a class of pure jump Lévy-type processes (the so-called stablelike processes), to [BC86], [Ish01], [KM14], [Kul07], [Mas07, Mas09], [KC99] and [Pic96, Pic10] for the case of a class of Lévy-type processes obtained as a solution of certain jump-type stochastic differential equations and [KS12] and [KS13] for the case of general Lévy-type processes.

As the first main result of this paper we prove that an open-set irreducible Lévy-type process $\left\{F_{t}\right\}_{t \geq 0}$ is transient if, and only if, there exist $x \in \mathbb{R}^{d}$ and an open bounded set $O \subseteq \mathbb{R}^{d}$, such that $L_{O}<\infty \mathbb{P}^{x}$-a.s. Accordingly, in the case of transience, for a Borel function $f:[0, \infty) \longrightarrow[0, \infty)$, we say that $\left\{F_{t}\right\}_{t \geq 0}$ is $f$-strongly transient if $\mathbb{E}^{x}\left[f\left(L_{O}\right)\right]<\infty$ for all $x \in \mathbb{R}^{d}$ and all open bounded sets $O \subseteq \mathbb{R}^{d}$. Otherwise, we say that $\left\{F_{t}\right\}_{t \geq 0}$ is $f$-weakly transient. Next, in the case when the function $f(t)$ is continuously differentiable and non-decreasing, we derive Chung-Fuchs type conditions for the $f$-weak and $f$-strong transience of $\left\{F_{t}\right\}_{t \geq 0}$. Specially, if $\left\{F_{t}\right\}_{t \geq 0}$ is a Lévy process, these conditions reduce to the Chung-Fuchs type conditions obtained in [SW04]. This shows that our conditions are sharp for Lévy processes. In the special case when $f(t)=t^{\kappa}$ for some $\kappa>0$, we use the terminology $\kappa$-weak and $\kappa$-strong transience introduced in [SW04]. In this context, we first discuss the $\kappa$-weak and $\kappa$-strong transience of $\left\{F_{t}\right\}_{t \geq 0}$ with respect to the dimension of the state space and Pruitt indices and, in particular, the $\kappa$-weak and $\kappa$-strong transience of elliptic diffusion and stable-like processes, thus generalizing the results obtained in [LL06] and [Tak67]. Finally, in the case when the symbol of $\left\{F_{t}\right\}_{t \geq 0}$ is radial in the co-variable we provide a series of conditions for the $\kappa$-weak and $\kappa$-strong transience in terms of the corresponding Lévy measure.

There is a vast literature and great efforts have been made to study the recurrence (and certain related properties such as null and positive recurrence, ergodicity and strong, subexponential and exponential ergodicity) of general and certain special subclasses of Markov processes. On the other hand, the transience property of Markov processes remained largely unexplored. The notion of weak and strong transience, as a "measure" of degree of transience, originated (and recognized to be crucial) from studying the asymptotic behavior as $t \longrightarrow \infty$ of the quantity $\int_{\mathbb{R}^{d}} \mathbb{P}^{x}\left(T_{B} \leq t\right) d x$ (the volume of a so-called Lévy sausage), where $B \in \mathcal{B}\left(\mathbb{R}^{d}\right)$ is bounded and $T_{B}:=\inf \{t \geq 0$ : $\left.L_{t} \in B\right\}$ is the first hitting time of $B$ by a transient Lévy process $\left\{L_{t}\right\}_{t \geq 0}$ (see [Por90] and the 
references therein). Except for Lévy processes, whose weak and strong transience have been studied extensively in [SW04] (see also [Haw77] for the case of symmetric Lévy processes and [SW05] and [Tak67] for the case of (semi-)stable Lévy processes), weak and strong transience of only few more special classes of Markov processes have been considered in the literature. More precisely, in [LL06] the authors have discussed weak and strong transience of elliptic diffusion processes. In [Yam98] weak and strong transience of Ornstein-Uhlenbeck type process (which are a subclass of Lévy-type processes with unbounded coefficients (see [SY84])) have been investigated and in [DGW05] the authors have considered weak and strong transience of a class of Lévy processes on metric Abelian groups. Finally, in [WZL06] strong transience of operator-self-similar Markov processes has been studied.

This paper is organized as follows. First, in Section 2, for a Borel function $f:[0, \infty) \longrightarrow[0, \infty)$, we introduce the notion of $f$-weak and $f$-strong transience of Lévy-type processes and derive ChungFuchs type conditions for these properties. In Section 3, we restrict to the $\kappa$-weak and $\kappa$-strong transience (that is, the case when $f(t)=t^{\kappa}$ for some $\kappa>0$ ) and discuss these properties with respect to the dimension of the state space and Pruitt indices. Finally, in Section 4, we consider $\kappa$-weak and $\kappa$-strong transience of Lévy-type processes with radial symbol (in the co-variable) and provide conditions for these properties in terms of the Lévy measures.

\section{Weak and Strong Transience of Lévy-Type Processes}

In this section, we introduce the notion and derive Chung-Fuchs type conditions for weak and strong transience of Lévy-type processes. We start with the following characterization of the transience property of Lévy-type processes.

Theorem 2.1. Let $\left\{F_{t}\right\}_{t \geq 0}$ be a d-dimensional open-set irreducible Lévy-type process. Then, the following properties are equivalent:

(i) $\left\{F_{t}\right\}_{t \geq 0}$ is transient;

(ii) there exists (for all) $x \in \mathbb{R}^{d}$ such that

$$
\mathbb{P}^{x}\left(\lim _{t \longrightarrow \infty}\left|F_{t}\right|=\infty\right)=1
$$

(iii) there exist (for all) $x \in \mathbb{R}^{d}$ and an (all) open bounded set $O \subseteq \mathbb{R}^{d}$, such that $L_{O}<\infty \mathbb{P}^{x}$-a.s.

Proof. (i) $\Leftrightarrow$ (ii) Let $\left\{\bar{F}_{n}\right\}_{n \geq 0}$ be a $d$-dimensional Markov chain generated by a transition function of the form

$$
\bar{p}(x, d y):=\int_{0}^{\infty} e^{-t} \mathbb{P}^{x}\left(F_{t} \in d y\right) d t .
$$

Clearly, $\left\{\bar{F}_{n}\right\}_{n \geq 0}$ is also open-set irreducible, and hence, due to [Twe94, Theorem 2.3], it is either transient or recurrent. Now, according to [TT79, Theorem 2.1] (which states that $\left\{\bar{F}_{n}\right\}_{n \geq 0}$ is transient if, and only if, $\left\{F_{t}\right\}_{t \geq 0}$ is transient) and [Sch98a, Corollary 3.4 and Theorem 5.2], [Twe94, Theorem 7.1] and [MT93, Proposition 3.2] (which state that the property in (ii) holds for $\left\{\bar{F}_{n}\right\}_{n \geq 0}$ if, and only if, it holds for $\left\{F_{t}\right\}_{t \geq 0}$ ), the assertion will follow if we prove that $\left\{\bar{F}_{n}\right\}_{n \geq 0}$ is transient if, and only if, there exists (for all) $x \in \mathbb{R}^{d}$ such that

$$
\overline{\mathbb{P}}^{x}\left(\lim _{n \longrightarrow \infty}\left|\bar{F}_{n}\right|=\infty\right)=1,
$$


where $\left\{\overline{\mathbb{P}}^{x}\right\}_{x \in \mathbb{R}^{d}}$ is induced by $\bar{p}(x, d y), x \in \mathbb{R}^{d}$. The necessity is a direct consequence of [MT09, Theorem 9.2.2 (i)] (together with [Sch98a, Corollary 3.4 and Theorem 5.2] and [MT09, Proposition 6.1.1 and Theorem 6.2.9]). On the other hand, the sufficiency is a consequence of (a straightforward generalization of) [San13, Proposition 5.3] and [MT09, Theorem 9.2.2 (ii)].

(i) $\Leftrightarrow$ (iii) First, recall that, due to the càdlàg property of the sample paths of $\left\{F_{t}\right\}_{t \geq 0}, L_{O}$ is a well-defined random variable. Now, the necessity follows directly from (ii). In order to conclude the sufficiency, observe that $\left\{\bar{F}_{n}\right\}_{n>0}$ (the chain defined in the first part of the proof) can be represented as follows. Let $\left\{J_{n}\right\}_{n>1}$ be a sequence of i.i.d. random variables with exponential distribution (with parameter one) defined on a probability space $(\bar{\Omega}, \overline{\mathcal{F}}, \overline{\mathbb{P}})$. Further, set $S_{0}:=0$ and $S_{n}:=J_{1}+\cdots+J_{n}, n \geq 1$. Then, $\left\{F_{S_{n}}\right\}_{n \geq 0}$ is a Markov chain defined on $\left(\Omega \times \bar{\Omega}, \mathcal{F} \times \overline{\mathcal{F}},\left\{\mathbb{P}^{x} \times \overline{\mathbb{P}}\right\}_{x \in \mathbb{R}^{d}}\right)$ having the same marginal distributions as $\left\{\bar{F}_{n}\right\}_{n \geq 0}$. Clearly, according to the strong law of large numbers, $\lim _{n \longrightarrow \infty} S_{n}=\infty \mathbb{P}$-a.s. Now, the assertion follows as a consequence of the transience and recurrence dichotomy of irreducible Markov chains and (a multidimensional version of) [San14b, Proposition 2.4].

Accordingly, in the sequel we always assume

(C4) $\left\{F_{t}\right\}_{t \geq 0}$ is a $d$-dimensional, open-set irreducible and transient Lévy-type process.

Now, we introduce the notion of weak and strong transience of Lévy-type processes. Let $f$ : $[0, \infty) \longrightarrow[0, \infty)$ be an arbitrary Borel measurable function. We say that $\left\{F_{t}\right\}_{t>0}$ is $f$-strongly transient if $\mathbb{E}^{x}\left[f\left(L_{O}\right)\right]<\infty$ for all $x \in \mathbb{R}^{d}$ and all open bounded sets $O \subseteq \mathbb{R}^{d}$. Otherwise, we say that $\left\{F_{t}\right\}_{t \geq 0}$ is $f$-weakly transient. In the following lemma we slightly generalize the result from [SW04, Lemma 2.2].

Lemma 2.2. Let $\left\{F_{t}\right\}_{t \geq 0}$ be a Lévy-type process satisfying condition (C4) and let $f:[0, \infty) \longrightarrow$ $[0, \infty)$ be a non-decreasing and continuously differentiable function. Then, for any $x \in \mathbb{R}^{d}$ and any open bounded sets $O_{1}, O_{2} \subseteq \mathbb{R}^{d}, \bar{O}_{1} \subseteq O_{2}$, there is a constant $c \geq 1$ such that

$$
c^{-1} \int_{0}^{\infty} f(t) \mathbb{P}^{x}\left(F_{t} \in \bar{O}_{1}\right) d t \leq \mathbb{E}^{x}\left[f\left(L_{\bar{O}_{1}}\right)\right] \leq c \int_{0}^{\infty} f(t) \mathbb{P}^{x}\left(F_{t} \in \bar{O}_{2}\right) d t .
$$

Here, $\bar{B}$ denotes the closure of the set $B \subseteq \mathbb{R}^{d}$.

Proof. Clearly, without loss of generality, we can assume that $f(0)=0$. Now, for any $x \in \mathbb{R}^{d}$ and any open set $O \subseteq \mathbb{R}^{d}$,

$$
\begin{aligned}
\mathbb{E}^{x}\left[f\left(L_{\bar{O}}\right)\right] & =\int_{[0, \infty)} f(t) d \mathbb{P}^{x}\left(L_{\bar{O}} \leq t\right) \\
& =\int_{[0, \infty)} \int_{[0, t)} f^{\prime}(s) d s d \mathbb{P}^{x}\left(L_{\bar{O}} \leq t\right) \\
& =\int_{0}^{\infty} f^{\prime}(t) \mathbb{P}^{x}\left(L_{\bar{O}}>t\right) d t \\
& =\int_{0}^{\infty} f^{\prime}(t) \mathbb{P}^{x}\left(T_{\bar{O}} \circ \theta_{t}<\infty\right) d t \\
& =\int_{0}^{\infty} f^{\prime}(t) \mathbb{E}^{x}\left[\mathbb{P}^{F_{t}}\left(T_{\bar{O}}<\infty\right)\right] d t
\end{aligned}
$$


where in the fourth step we used the fact $\left\{L_{\bar{O}}>t\right\}=\left\{T_{\bar{O}} \circ \theta_{t}<\infty\right\}$. Recall that $T_{B}$ denotes the first hitting time of the set $B \subseteq \mathbb{R}^{d}$ by $\left\{F_{t}\right\}_{t \geq 0}$. Further, [Yam98, Lemma 2.2] states that if

$$
\inf _{x \in K} \mathbb{E}^{x}\left[e^{-L_{K}}\right]>0 \quad \text { for every compact set } K \subseteq \mathbb{R}^{d},
$$

then for any $x \in \mathbb{R}^{d}$ and any open bounded sets $O_{1}, O_{2} \subseteq \mathbb{R}^{d}, \bar{O}_{1} \subseteq O_{2}$, there is a constant $c \geq 1$ such that

$$
c^{-1} \int_{0}^{\infty} \int_{0}^{\infty} \mathbb{E}^{x}\left[\mathbb{P}^{F_{t}}\left(F_{s} \in \bar{O}_{1}\right)\right] d s f^{\prime}(t) d t \leq \mathbb{E}^{x}\left[f\left(L_{\bar{O}_{1}}\right)\right] \leq c \int_{0}^{\infty} \int_{0}^{\infty} \mathbb{E}^{x}\left[\mathbb{P}^{F_{t}}\left(F_{s} \in \bar{O}_{2}\right)\right] d s f^{\prime}(t) d t .
$$

On the other hand, for any $x \in \mathbb{R}^{d}$ and any open set $O \subseteq \mathbb{R}^{d}$,

$$
\int_{0}^{\infty} \int_{0}^{\infty} \mathbb{E}^{x}\left[\mathbb{P}^{F_{t}}\left(F_{s} \in \bar{O}\right)\right] d s f^{\prime}(t) d t=\int_{0}^{\infty} \mathbb{E}^{x}\left[\int_{t}^{\infty} 1_{\bar{O}}\left(F_{s}\right) d s\right] f^{\prime}(t) d t=\int_{0}^{\infty} f(t) \mathbb{P}^{x}\left(F_{t} \in \bar{O}\right) d t
$$

which proves the claim. Finally, let us prove the relation in (2.1). Let $K \subseteq \mathbb{R}^{d}$ be an arbitrary compact set. First, observe that, by the Markov property,

$$
\mathbb{E}^{x}\left[e^{-L_{K}}\right] \geq e^{-t} \int_{\mathbb{R}^{d}} \mathbb{E}^{y}\left[e^{-L_{K}}\right] \mathbb{P}^{x}\left(F_{t} \in d y\right), \quad x \in \mathbb{R}^{d}, t \geq 0 .
$$

Further, according to [Sch98a, Corollary 3.4 and Theorem 5.2] and [Twe94, Theorem 7.1], there exist a probability measure $a(d t)$ on $\mathcal{B}([0, \infty))$ and a Borel kernel $T: \mathbb{R}^{d} \times \mathcal{B}\left(\mathbb{R}^{d}\right) \longrightarrow[0,1]$, such that

(i) $T\left(x, \mathbb{R}^{d}\right)>0$ for all $x \in \mathbb{R}^{d}$;

(ii) $\int_{[0, \infty)} \mathbb{P}^{x}\left(F_{t} \in B\right) a(d t) \geq T(x, B)$ for all $x \in \mathbb{R}^{d}$ and all $B \in \mathcal{B}\left(\mathbb{R}^{d}\right)$;

(iii) $\liminf _{y \longrightarrow x} \int_{\mathbb{R}^{d}} f(z) T(y, d z) \geq \int_{\mathbb{R}^{d}} f(z) T(x, d z)$ for all $x \in \mathbb{R}^{d}$ and all $f \in B_{b}\left(\mathbb{R}^{d}\right), f \geq 0$.

Now, fix $t_{0}>0$. Then, from the previous relation we have

$$
\begin{aligned}
& \mathbb{E}^{x}\left[e^{-L_{K}}\right] \int_{\left[0, t_{0}\right]} a(d t) \\
& \geq e^{-t_{0}} \int_{\left[0, t_{0}\right]} \int_{\mathbb{R}^{d}} \mathbb{E}^{y}\left[e^{-L_{K}}\right] \mathbb{P}^{x}\left(F_{t} \in d y\right) a(d t) \\
& =e^{-t_{0}} \int_{[0, \infty)} \int_{\mathbb{R}^{d}} \mathbb{E}^{y}\left[e^{-L_{K}}\right] \mathbb{P}^{x}\left(F_{t} \in d y\right) a(d t)-e^{-t_{0}} \int_{\left(t_{0}, \infty\right)} \int_{\mathbb{R}^{d}} \mathbb{E}^{y}\left[e^{-L_{K}}\right] \mathbb{P}^{x}\left(F_{t} \in d y\right) a(d t) \\
& \geq e^{-t_{0}} \int_{\mathbb{R}^{d}} \mathbb{E}^{y}\left[e^{-L_{K}}\right] T(x, d y)-e^{-t_{0}} \int_{\left(t_{0}, \infty\right)} a(d t) .
\end{aligned}
$$

Hence, if (2.1) did not hold for $K$, then we would have

$$
\int_{\left(t_{0}, \infty\right)} a(d t) \geq \inf _{x \in K} \int_{\mathbb{R}^{d}} \mathbb{E}^{y}\left[e^{-L_{K}}\right] T(x, d y),
$$

that is, by letting $t_{0} \longrightarrow \infty$,

$$
\inf _{x \in K} \int_{\mathbb{R}^{d}} \mathbb{E}^{y}\left[e^{-L_{K}}\right] T(x, d y)=0 .
$$


In particular, due to the compactness of $K$, there would exist a sequence $\left\{x_{n}\right\}_{n \geq 1} \subseteq K, \lim _{n \longrightarrow \infty} x_{n}=$ $x_{0} \in K$, such that

$$
0=\lim _{n \longrightarrow \infty} \int_{\mathbb{R}^{d}} \mathbb{E}^{y}\left[e^{-L_{K}}\right] T\left(x_{n}, d y\right) \geq \liminf _{x \longrightarrow x_{0}} \int_{\mathbb{R}^{d}} \mathbb{E}^{y}\left[e^{-L_{K}}\right] T(x, d y) \geq \int_{\mathbb{R}^{d}} \mathbb{E}^{y}\left[e^{-L_{K}}\right] T\left(x_{0}, d y\right) .
$$

However, this cannot be the case since $T\left(x_{0}, \mathbb{R}^{d}\right)>0$ and, due to the transience of $\left\{F_{t}\right\}_{t \geq 0}$, $\mathbb{E}^{x}\left[e^{-L_{K}}\right]>0$ for all $x \in \mathbb{R}^{d}$.

Let us remark that if the semigroup $\left\{P_{t}\right\}_{t \geq 0}$ of $\left\{F_{t}\right\}_{t \geq 0}$ satisfies the strong Feller property, that is, $P_{t}\left(B_{b}\left(\mathbb{R}^{d}\right)\right) \subseteq C\left(\mathbb{R}^{d}\right)$ for all $t>0$, then Theorem 2.1 and the relation in (2.1) follow directly from (a multidimensional version of) [San14b, Proposition 2.4] and [Yam98, Remark 2.3], respectively. For sufficient conditions for a Feller semigroup to be a strong Feller semigroup see [SW12] and [SW13]. In the following two theorems we derive Chung-Fuchs type conditions for the $f$-weak and $f$-strong transience.

Theorem 2.3. Let $f:[0, \infty) \longrightarrow[0, \infty)$ be a non-decreasing and continuously differentiable function and let $\left\{F_{t}\right\}_{t \geq 0}$ be a Lévy-type process with infinitesimal generator $\left(\mathcal{A}, \mathcal{D}_{\mathcal{A}}\right)$ and symbol $q(x, \xi)$, satisfying condition (C4). Assume

$$
\liminf _{\alpha \longrightarrow 0} \int_{\mathbb{R}^{d}}\left(\int_{\frac{\ln 2}{4 \sup _{x \in \mathbb{R}^{d}}|q(x, \xi)|}}^{\infty} e^{-\alpha t} f(t) \operatorname{Re} \mathbb{E}^{0}\left[e^{i\left\langle\xi, F_{t}\right\rangle}\right] d t\right) \frac{\sin ^{2}\left(\frac{a \xi_{1}}{2}\right)}{\xi_{1}^{2}} \cdots \frac{\sin ^{2}\left(\frac{a \xi_{d}}{2}\right)}{\xi_{d}^{2}} d \xi>-\infty
$$

for all $a>0$ small enough. Then, $\left\{F_{t}\right\}_{t \geq 0}$ is $f$-weakly transient if

$$
\int_{B(0, r)} \int_{0}^{\frac{\ln 2}{4 \sup _{x \in \mathbb{R}^{d}}|q(x, \xi)|}} f(t) d t d \xi=\infty \quad \text { for some } r>0 .
$$

Proof. We follow the proof of [San14a, Theorem 1.2] and prove that, under the above assumptions, $\mathbb{E}^{0}\left[L_{O_{0}}\right]=\infty$ for every open neighborhood $O_{0} \subseteq \mathbb{R}^{d}$ of the origin. According to Lemma 2.2, it suffices to prove that

$$
\int_{0}^{\infty} f(t) \mathbb{P}^{0}\left(F_{t} \in O_{0}\right) d t=\infty
$$

for every open neighborhood $O_{0} \subseteq \mathbb{R}^{d}$ of the origin. Let $a>0$ be arbitrary. By the monotone convergence theorem, we have

$$
\begin{aligned}
\int_{0}^{\infty} f(t) \mathbb{P}^{0}\left(F_{t} \in(-a, a)^{d}\right) d t & =\lim _{\alpha \longrightarrow 0} \mathbb{E}^{0}\left[\int_{0}^{\infty} e^{-\alpha t} f(t) 1_{\left\{F_{t} \in(-a, a)^{d}\right\}} d t\right] \\
& =\lim _{\alpha \longrightarrow 0} \int_{0}^{\infty} \int_{\mathbb{R}^{d}} e^{-\alpha t} f(t) 1_{(-a, a)^{d}}(y) \mathbb{P}^{0}\left(F_{t} \in d y\right) d t
\end{aligned}
$$

where $(-a, a)^{d}:=(-a, a) \times \ldots \times(-a, a)$. Next, let

$$
g(u):=\left(1-\frac{|u|}{a}\right) 1_{(-a, a)}(u), \quad u \in \mathbb{R}
$$

and

$$
h(y):=g\left(y_{1}\right) \cdots g\left(y_{d}\right), \quad y=\left(y_{1}, \ldots, y_{d}\right) \in \mathbb{R}^{d} .
$$

Clearly,

$$
1_{(-a, a)^{d}}(y) \geq h(y), \quad y \in \mathbb{R}^{d} .
$$


According to this,

$$
\int_{0}^{\infty} f(t) \mathbb{P}^{0}\left(F_{t} \in(-a, a)^{d}\right) d t \geq \liminf _{\alpha \longrightarrow 0} \int_{0}^{\infty} \int_{\mathbb{R}^{d}} e^{-\alpha t} f(t) h(y) \mathbb{P}^{0}\left(F_{t} \in d y\right) d t .
$$

Further, observe that

$$
g(u)=\frac{1}{\sqrt{a}} 1_{\left(-\frac{a}{2}, \frac{a}{2}\right)} * \frac{1}{\sqrt{a}} 1_{\left(-\frac{a}{2}, \frac{a}{2}\right)}(u), \quad u \in \mathbb{R},
$$

where $*$ denotes the standard convolution operator. Hence, since

$$
\mathcal{F}\left(\frac{1}{\sqrt{a}} 1_{\left(-\frac{a}{2}, \frac{a}{2}\right)}\right)(\xi)=\frac{\sin \left(\frac{a \xi}{2}\right)}{\sqrt{a} \pi \xi},
$$

we have

$$
\mathcal{F}(h)(\xi)=\frac{\sin ^{2}\left(\frac{a \xi_{1}}{2}\right)}{a \pi^{2} \xi_{1}^{2}} \cdots \frac{\sin ^{2}\left(\frac{a \xi_{d}}{2}\right)}{a \pi^{2} \xi_{d}^{2}} .
$$

Denote by $\Phi_{t}(x, \xi):=\mathbb{E}^{x}\left[e^{i\left\langle\xi, F_{t}-x\right\rangle}\right]$ for $t \geq 0$ and $x, \xi \in \mathbb{R}^{d}$. The above facts yield

$$
\begin{aligned}
\int_{0}^{\infty} f(t) \mathbb{P}^{0}\left(F_{t} \in(-a, a)^{d}\right) d t & \geq \liminf _{\alpha \longrightarrow 0} \int_{0}^{\infty} \int_{\mathbb{R}^{d}} \int_{\mathbb{R}^{d}} e^{-\alpha t} f(t) e^{i\langle\xi, y\rangle} \mathcal{F}(h)(\xi) d \xi \mathbb{P}^{0}\left(F_{t} \in d y\right) d t \\
& =\liminf _{\alpha \longrightarrow 0} \int_{0}^{\infty} \int_{\mathbb{R}^{d}} e^{-\alpha t} f(t) \Phi_{t}(0, \xi) \mathcal{F}(h)(\xi) d \xi d t \\
& =\liminf _{\alpha \longrightarrow 0} \int_{0}^{\infty} \int_{\mathbb{R}^{d}} e^{-\alpha t} f(t) \operatorname{Re} \Phi_{t}(0, \xi) \mathcal{F}(h)(\xi) d \xi d t .
\end{aligned}
$$

Now, (the proof of) [San14a, Lemma 2.2] states that

$$
\operatorname{Re} \Phi_{t}(0, \xi) \geq \exp \left[-4 t \sup _{x \in \mathbb{R}^{d}}|q(x, \xi)|\right], \quad t \in\left[0, \frac{\ln 2}{4 \sup _{x \in \mathbb{R}^{d}}|q(x, \xi)|}\right] .
$$

Hence,

$$
\begin{aligned}
& \int_{0}^{\infty} f(t) \mathbb{P}^{0}\left(F_{t} \in(-a, a)^{d}\right) d t \\
& \geq \liminf _{\alpha \longrightarrow 0} \int_{\mathbb{R}^{d}}\left(\int_{0}^{t_{0}(\xi)} e^{-\alpha t} f(t) \operatorname{Re} \Phi_{t}(0, \xi) d t+\int_{t_{0}(\xi)}^{\infty} e^{-\alpha t} f(t) \operatorname{Re} \Phi_{t}(0, \xi) d t\right) \mathcal{F}(h)(\xi) d \xi \\
& \geq \liminf _{\alpha \longrightarrow 0} \int_{\mathbb{R}^{d}}\left(\int_{0}^{t_{0}(\xi)} f(t) d t\right) \exp \left[-t_{0}(\xi)\left(\alpha+4 \sup _{x \in \mathbb{R}^{d}}|q(x, \xi)|\right)\right] \mathcal{F}(h)(\xi) d \xi \\
& \quad+\liminf _{\alpha \longrightarrow 0} \int_{\mathbb{R}^{d}} \int_{t_{0}(\xi)}^{\infty} e^{-\alpha t} f(t) \operatorname{Re} \Phi_{t}(0, \xi) d t \mathcal{F}(h)(\xi) d \xi,
\end{aligned}
$$

where $t_{0}(\xi):=\ln 2 / 4 \sup _{x \in \mathbb{R}^{d}}|q(x, \xi)|$. Consequently, by the monotone convergence theorem, we have

$$
\begin{aligned}
& \int_{0}^{\infty} f(t) \mathbb{P}^{0}\left(F_{t} \in(-a, a)^{d}\right) d t \\
& \geq \frac{1}{2} \int_{\mathbb{R}^{d}}\left(\int_{0}^{t_{0}(\xi)} f(t) d t\right) \mathcal{F}(h)(\xi) d \xi+\liminf _{\alpha \longrightarrow 0} \int_{\mathbb{R}^{d}} \int_{t_{0}(\xi)}^{\infty} e^{-\alpha t} f(t) \operatorname{Re} \Phi_{t}(0, \xi) d t \mathcal{F}(h)(\xi) d \xi .
\end{aligned}
$$


Finally, let $r>0$ be such that

$$
\int_{B(0, r)} \int_{0}^{t_{0}(\xi)} f(t) d t d \xi=\infty
$$

Then, since

$$
\lim _{a \longrightarrow 0} \frac{(2 \pi)^{2 d}}{a^{d}} \mathcal{F}(h)(\xi)=1,
$$

for any $c \in(0,1)$, all $\xi \in B(0, r)$ and all $a>0$ small enough we have

$$
\begin{aligned}
& \int_{0}^{\infty} f(t) \mathbb{P}^{0}\left(F_{t} \in(-a, a)^{d}\right) d t \\
& \geq \frac{c a^{d}}{2(2 \pi)^{2 d}} \int_{B(0, r)} \int_{0}^{t_{0}(\xi)} f(t) d t d \xi+\liminf _{\alpha \longrightarrow 0} \int_{\mathbb{R}^{d}} \int_{t_{0}(\xi)}^{\infty} e^{-\alpha t} f(t) \operatorname{Re} \Phi_{t}(0, \xi) d t \mathcal{F}(h)(\xi) d \xi,
\end{aligned}
$$

which together with (2.2) concludes the proof.

Proposition 2.4. Let $\left\{F_{t}\right\}_{t \geq 0}$ be a Lévy-type process with infinitesimal generator $\left(\mathcal{A}, \mathcal{D}_{\mathcal{A}}\right)$ and symbol $q(x, \xi)$. Assume that

(i) $C_{c}^{\infty}\left(\mathbb{R}^{d}\right)$ is an operator core for $\left(\mathcal{A}, \mathcal{D}_{\mathcal{A}}\right)$, that is, $\mathcal{A}$ is the unique extension of $\left.\mathcal{A}\right|_{C_{c}^{\infty}\left(\mathbb{R}^{d}\right)}$ on $\mathcal{D}_{\mathcal{A}}$

(ii) $q(x, \xi)=q(-x,-\xi)$ for all $x, \xi \in \mathbb{R}^{d}$.

Then, $\Phi_{t}(0, \xi)=\operatorname{Re} \Phi_{t}(0, \xi)$ for all $t \geq 0$ and all $\xi \in \mathbb{R}^{d}$.

Proof. Define $\bar{F}_{t}:=-F_{t}$ for $t \geq 0$. It is straightforward to check that the process $\left(\left\{\bar{F}_{t}\right\}_{t \geq 0},\left\{\overline{\mathbb{P}}^{x}\right\}_{x \in \mathbb{R}^{d}}\right)$, where $\overline{\mathbb{P}}^{x}\left(\bar{F}_{t} \in d y\right):=\mathbb{P}^{-x}\left(-F_{t} \in d y\right)$ for $t \geq 0$ and $x \in \mathbb{R}^{d}$, is a Feller process with symbol $\bar{q}(x, \xi):=q(-x,-\xi)$ for $x, \xi \in \mathbb{R}^{d}$. Furthermore, since $C_{c}^{\infty}\left(\mathbb{R}^{d}\right)$ is an operator core for $\left(\mathcal{A}, \mathcal{D}_{\mathcal{A}}\right)$ and, by assumption, $\bar{q}(x, \xi)=q(x, \xi)$ for all $x, \xi \in \mathbb{R}^{d}$, [EK86, Proposition 4.1.7] and [SW13, Proposition 4.6] state that $\left(\left\{F_{t}\right\}_{t \geq 0},\left\{\mathbb{P}^{x}\right\}_{x \in \mathbb{R}^{d}}\right)$ and $\left(\left\{\bar{F}_{t}\right\}_{t \geq 0},\left\{\overline{\mathbb{P}}^{x}\right\}_{x \in \mathbb{R}^{d}}\right)$ have the same finite-dimensional distributions. In particular, for any $t \geq 0$ and any $x, \xi \in \mathbb{R}^{d}$,

$$
\Phi_{t}(x, \xi)=\mathbb{E}^{x}\left[e^{i\left\langle F_{t}-x, \xi\right\rangle}\right]=\overline{\mathbb{E}}^{x}\left[e^{i\left\langle\bar{F}_{t}-x, \xi\right\rangle}\right]=\mathbb{E}^{-x}\left[e^{i\left\langle-F_{t}+x, \xi\right\rangle}\right]=\mathbb{E}^{-x}\left[e^{i\left\langle F_{t}-x,-\xi\right\rangle}\right]=\Phi_{t}(-x,-\xi),
$$

which proves the assertion.

Observe that Lévy processes and elliptic diffusion processes always satisfy (i). Also, many jump Lévy-type processes, like stable-like processes (see Example 3.5), Lévy-type processes generated by finite Lévy measures (see Example 4.8) and Lévy-type processes with sufficiently regular symbol (see [BSW13, Theorem 3.23]), have this property. The condition in (ii) implies (actually it is equivalent) that the corresponding Lévy triplet $(b(x), C(x), \nu(x, d y))$ satisfies: $b(x)=-b(-x), C(x)=C(-x)$ and $\nu(x, d y)=\nu(-x,-d y)$ for all $x \in \mathbb{R}^{d}$.

The condition in (2.2) is a crucial assumption in Theorem 2.3. Clearly, it trivially holds true in the case of a symmetric Lévy process. Moreover, in that case the left hand side in (2.2) is nonnegative. We conjecture that the same property should hold in the case of a Lévy-type process satisfying the assumptions from Proposition 2.4. Directly from Bochner's theorem (see [Jac01, Theorem 3.5.7]) we get the following.

Proposition 2.5. Let $\left\{F_{t}\right\}_{t \geq 0}$ be a Lévy-type process which admits a transition density function $p(t, x, y), t>0, x, y \in \mathbb{R}^{d}$, satisfying: 
(i) $\int_{\mathbb{R}^{d}}\left|\Phi_{t}(0, \xi)\right| d \xi<\infty$ for all $t>0$;

(ii) the function $y \longmapsto p(t, 0, y)$ is continuous for all $t>0$;

(iii) the function $y \longmapsto p(t, 0, y)$ is positive definite for all $t>0$, that is, for all $n \in \mathbb{N}$, all $y_{1}, \ldots, y_{n} \in \mathbb{R}^{d}$, all $c_{1}, \ldots, c_{n} \in \mathbb{C}$ and all $t>0$,

$$
\sum_{i=1}^{n} \sum_{j=1}^{n} c_{i} \bar{c}_{j} p\left(t, 0, y_{i}-y_{j}\right) \geq 0 .
$$

Then, $\Phi_{t}(0, \xi) \geq 0$ for all $t \geq 0$ and all $\xi \in \mathbb{R}^{d}$.

Let us remark that the existence of a transition density function and (i) follow if

$$
\int_{\mathbb{R}^{d}} \exp \left[-t \inf _{x \in \mathbb{R}^{d}} \operatorname{Re} q(x, \xi)\right] d \xi<\infty, \quad t>0
$$

and (2.4) hold (see [SW13, Theorem 2.7]). Moreover, under the same assumptions, (ii) follows as a direct consequence of the dominated convergence theorem. On the other hand, (iii) holds if the functions $y \longmapsto p(t, 0, y)$ is symmetric (which is the case under the assumptions of Proposition 2.4) and $p(t, 0,0)=\sup _{y \in \mathbb{R}^{d}} p(t, 0, y)$ for all $t>0$.

Theorem 2.6. Let $f:[0, \infty) \longrightarrow[0, \infty)$ be a non-decreasing and continuously differentiable function and let $\left\{F_{t}\right\}_{t \geq 0}$ be a Lévy-type process with symbol $q(x, \xi)$, satisfying condition (C4). Then, $\left\{F_{t}\right\}_{t \geq 0}$ is $f$-strongly transient if there exists a constant $0 \leq c<1$ such that

$$
\sup _{x \in \mathbb{R}^{d}}|\operatorname{Im} q(x, \xi)| \leq c \inf _{x \in \mathbb{R}^{d}} \operatorname{Re} q(x, \xi), \quad \xi \in \mathbb{R}^{d},
$$

and

$$
\int_{B(0, r)} \int_{0}^{\infty} f(t) \exp \left[-\frac{t}{16} \inf _{x \in \mathbb{R}^{d}} \operatorname{Re} q(x, \xi)\right] d t d \xi<\infty \quad \text { for some } r>0 .
$$

Proof. We follow the proof of [SW13, Theorem 1.1] and prove that, under the above assumptions, $\mathbb{E}^{x}\left[L_{O_{x}}\right]<\infty$ for all $x \in \mathbb{R}^{d}$ and all open bounded neighborhoods $O_{x} \subseteq \mathbb{R}^{d}$ of $x$. According to Lemma 2.2, it suffices to prove that

$$
\int_{0}^{\infty} f(t) \mathbb{P}^{x}\left(F_{t} \in O_{x}\right) d t<\infty
$$

for all $x \in \mathbb{R}^{d}$ and all open bounded neighborhoods $O_{x} \subseteq \mathbb{R}^{d}$ of $x$. We proceed, and use the same notation, as in the proof of Theorem 2.3. Fix $x=\left(x_{1}, \ldots, x_{d}\right) \in \mathbb{R}^{d}$ and $a>0$. Define

$$
g_{j}(u):=e^{i x_{j} u}\left(1-\frac{|u|}{a}\right) 1_{(-a, a)}(u), \quad u \in \mathbb{R}, j=1, \ldots, d,
$$

and

$$
h(y):=g_{1}\left(y_{1}\right) \cdots g\left(y_{d}\right), \quad y=\left(y_{1}, \ldots, y_{d}\right) \in \mathbb{R}^{d} .
$$

Next, since

$$
\mathcal{F}^{-1}\left(\frac{\sin ^{2}\left(\frac{a\left(\cdot-x_{j}\right)}{2}\right)}{a \pi^{2}\left(\cdot-x_{j}\right)^{2}}\right)(u)=g_{j}(u), \quad u \in \mathbb{R}, j=1, \ldots, d,
$$


we conclude

$$
\mathcal{F}(h)(\xi)=\frac{\sin ^{2}\left(\frac{a\left(\xi_{1}-x_{1}\right)}{2}\right)}{a \pi^{2}\left(\xi_{1}-x_{1}\right)^{2}} \cdots \frac{\sin ^{2}\left(\frac{a\left(\xi_{d}-x_{d}\right)}{2}\right)}{a \pi^{2}\left(\xi_{d}-x_{d}\right)^{2}}, \quad \xi=\left(\xi_{1}, \ldots, \xi_{d}\right) \in \mathbb{R}^{d} .
$$

Here, $\mathcal{F}^{-1}$ denotes the inverse Fourier transform. Now, since $\sin u / u \geq 1 / 2$ for all $|u| \leq \pi / 3$, we have

$$
\mathcal{F}(h)(\xi) \geq \frac{a^{d}}{4^{d} \pi^{2 d}}, \quad \xi \in(x-2 \pi / 3 a, x+2 \pi / 3 a)^{d},
$$

where $(x-2 \pi / 3 a, x+2 \pi / 3 a)^{d}:=\left(x_{1}-2 \pi / 3 a, x_{1}+2 \pi / 3 a\right) \times \cdots \times\left(x_{d}-2 \pi / 3 a, x_{d}+2 \pi / 3 a\right)$. According to this and the monotone convergence theorem,

$$
\frac{a^{d}}{8^{d} \pi^{2 d}} \int_{0}^{\infty} f(t) \mathbb{P}^{x}\left(F_{t} \in(x-2 \pi / 3 a, x+2 \pi / 3 a)^{d}\right) d t \leq \lim _{\alpha \longrightarrow 0} \int_{0}^{\infty} e^{-\alpha t} f(t) \mathbb{E}^{x}\left[\mathcal{F}(h)\left(F_{t}\right)\right] d t .
$$

Further, [Jac98, Theorem 1.1] implies that

$$
\mathbb{E}^{x}\left[\mathcal{F}(h)\left(F_{t}\right)\right]=\frac{1}{(2 \pi)^{d}} \int_{\mathbb{R}^{d}} e^{-i\langle x, y\rangle} h(y) \Phi_{t}(x, y) d y, \quad t \geq 0,
$$

which yields

$$
\begin{aligned}
& \frac{a^{d}}{(4 \pi)^{d}} \int_{0}^{\infty} f(t) \mathbb{P}^{x}\left(F_{t} \in(x-2 \pi / 3 a, x+2 \pi / 3 a)^{d}\right) d t \\
& \leq \lim _{\alpha \longrightarrow 0} \int_{0}^{\infty} \int_{\mathbb{R}^{d}} e^{-\alpha t} f(t) e^{-i\langle x, y\rangle} h(y) \Phi_{t}(x, y) d y d t \\
& =\lim _{\alpha \longrightarrow 0} \int_{0}^{\infty} \int_{\mathbb{R}^{d}} e^{-\alpha t} f(t) e^{-i\langle x, y\rangle} h(y) \operatorname{Re} \Phi_{t}(x, y) d y d t \\
& \leq \lim _{\alpha \longrightarrow 0} \int_{0}^{\infty} \int_{\mathbb{R}^{d}} e^{-\alpha t} f(t) e^{-i\langle x, y\rangle} h(y)\left|\Phi_{t}(x, y)\right| d y d t \\
& \leq \lim _{\alpha \longrightarrow 0} \int_{0}^{\infty} \int_{(-a, a)^{d}} e^{-\alpha t} f(t)\left|\Phi_{t}(x, y)\right| d y d t \\
& \leq \lim _{\alpha \longrightarrow 0} \int_{0}^{\infty} \int_{B(0, a \sqrt{d})} e^{-\alpha t} f(t)\left|\Phi_{t}(x, y)\right| d y d t .
\end{aligned}
$$

Recall that $\Phi_{t}(x, y)=\mathbb{E}^{x}\left[e^{i\left\langle y, F_{t}-x\right\rangle}\right]$ for $t \geq 0$ and $x, y \in \mathbb{R}^{d}$. Now, due to [SW13, Theorem 2.7], under (2.4) we have that

$$
\left|\Phi_{t}(x, y)\right| \leq \exp \left[-\frac{t}{16} \inf _{z \in \mathbb{R}^{d}} \operatorname{Re} q(z, 2 y)\right], \quad t \geq 0, y \in \mathbb{R}^{d} .
$$

Thus,

$$
\begin{aligned}
& \int_{0}^{\infty} f(t) \mathbb{P}^{x}\left(F_{t} \in(x-2 \pi / 3 a, x+2 \pi / 3 a)^{d}\right) d t \\
& \leq \frac{(4 \pi)^{d}}{a^{d}} \int_{B(0, a \sqrt{d})} \int_{0}^{\infty} f(t) \exp \left[-\frac{t}{16} \inf _{x \in \mathbb{R}^{d}} \operatorname{Re} q(x, 2 y)\right] d t d y \\
& =\frac{(2 \pi)^{d}}{a^{d}} \int_{B(0,2 a \sqrt{d})} \int_{0}^{\infty} f(t) \exp \left[-\frac{t}{16} \inf _{x \in \mathbb{R}^{d}} \operatorname{Re} q(x, y)\right] d t d y,
\end{aligned}
$$

which completes the proof. 
Let us remark that when $f(t)$ is a constant function, then the conditions in $(2.3)$ and $(2.5)$ become Chung-Fuchs type conditions for recurrence and transience, respectively, derived in [San14a, Theorem 1.2] and [SW13, Theorem 1.1]. Further, observe that if the condition in (2.3) holds for some $r_{0}>0$, then it also holds for all $r \geq r_{0}$. On the other hand, if (2.5) holds for some $r_{0}>0$, than it also holds for all $r \leq r_{0}$. Clearly, if we assume that

$$
\inf _{r \leq|\xi| \leq r_{0}} \sup _{x \in \mathbb{R}^{d}}|q(x, \xi)|>0, \quad 0<r<r_{0}
$$

then (2.3) does not depend on $r>0$. Recall, a function $f: \mathbb{R}^{d} \longrightarrow \mathbb{R}$ is said to be radial if for any orthogonal $d \times d$ matrix $O, f(x)=f(O x)$ for all $x \in \mathbb{R}^{d}$.

Proposition 2.7. Let $\left\{F_{t}\right\}_{t \geq 0}$ be a Lévy-type process with symbol $q(x, \xi)$. If the function $\xi \longmapsto$ $\sup _{x \in \mathbb{R}^{d}}|q(x, \xi)|$ is radial, then the condition in (2.3) does not depend on $r>0$.

Proof. Let $r_{0}>0$ be such that

$$
\int_{B\left(0, r_{0}\right)} \int_{0}^{\frac{\ln 2}{4 \sup _{x \in \mathbb{R}^{d}}|q(x, \xi)|}} f(t) d t d \xi<\infty
$$

and

$$
\int_{B(0, r)} \int_{0}^{\frac{\ln 2}{4 \sup _{x \in \mathbb{R}^{d}}|q(x, \xi)|}} f(t) d t d \xi=\infty, \quad r>r_{0}
$$

In particular,

$$
\int_{B(0, r) \backslash B\left(0, r_{0}\right)} \int_{0}^{\frac{\ln 2}{4 \sup _{x \in \mathbb{R}^{d}}|q(x, \xi)|}} f(t) d t d \xi=\infty, \quad r>r_{0} .
$$

Now, according to (the proof of) [San14a, Proposition 2.4], the above relation (together with the radiality property of the function $\left.\xi \longmapsto \sup _{x \in \mathbb{R}^{d}}|q(x, \xi)|\right)$ implies that for any $\xi_{0} \in \mathbb{R}^{d},\left|\xi_{0}\right|=r_{0}$, the function $\xi \longmapsto \sup _{x \in \mathbb{R}^{d}}|q(x, \xi)|$ is continuous and periodic with period $\xi_{0}$. Consequently, $q(x, \xi)=0$ for all $x, \xi \in \mathbb{R}^{d}$, which is impossible.

Finally, recall that if $\left\{L_{t}\right\}_{t \geq 0}$ is a symmetric Lévy process (that is, $\left\{L_{t}\right\}_{t \geq 0} \stackrel{\mathrm{d}}{=}\left\{-L_{t}\right\}_{t \geq 0}$ ), then $q(\xi)=\operatorname{Re} q(\xi)$ and $\mathbb{E}^{x}\left[e^{i\left\langle\xi, L_{t}-x\right\rangle}\right]=\operatorname{Re} \mathbb{E}^{x}\left[e^{i\left\langle\xi, L_{t}-x\right\rangle}\right]=e^{-t q(\xi)}>0$ for all $t \geq 0$ and all $x, \xi \in \mathbb{R}^{d}$. Hence, if, in addition, $\left\{L_{t}\right\}_{t>0}$ is open-set irreducible, Theorems 2.3 and 2.6 apply. However, as we commented in the first section, because of the stationarity and independence of the increments, the assumptions on the open-set irreducibility is not needed in order to introduce the notion of the $f$-weak and $f$-strong transience, and therefore to derive Chung-Fuchs type conditions for these properties, of Lévy processes. By completely the same reasoning as in the proofs of Theorems 2.3 and 2.6 we easily obtain the following criterion for the $f$-weak and $f$-strong transience of symmetric Lévy processes (see also [SW04, Theorem 3.4]).

Corollary 2.8. Let $f:[0, \infty) \longrightarrow[0, \infty)$ be a non-decreasing and continuously differentiable function. Then, a symmetric and transient Lévy process $\left\{L_{t}\right\}_{t \geq 0}$ with symbol $q(\xi)$ is $f$-weakly transient if, and only if,

$$
\int_{B(0, r)} \int_{0}^{\infty} f(t) e^{-t q(\xi)} d t d \xi=\infty \quad \text { for some (all) } r>0 .
$$




\section{Algebraic Weak and Strong Transience of Lévy-Type Processes}

In this section, we concentrate on the $f$-weak and $f$-strong transience with respect to $f(t):=t^{\kappa}$ for some $\kappa>0$ and we use the terminology $\kappa$-weak and $\kappa$-strong transience, respectively. Obviously, the Chung-Fuchs type conditions derived in Theorems 2.3 and 2.6 now read

$$
\begin{gathered}
\int_{B(0, r)} \frac{d \xi}{\left(\sup _{x \in \mathbb{R}^{d}}|q(x, \xi)|\right)^{\kappa+1}}=\infty \quad \text { for some } r>0 \\
\int_{B(0, r)} \frac{d \xi}{\left(\inf _{x \in \mathbb{R}^{d}} \operatorname{Re} q(x, \xi)\right)^{\kappa+1}}<\infty \quad \text { for some } r>0
\end{gathered}
$$

respectively. As a simple consequence, we also get the following Chung-Fuchs type condition for the $\kappa$-strong transience.

Corollary 3.1. Let $\kappa>0$ and let $\left\{F_{t}\right\}_{t \geq 0}$ be a Lévy-type process with symbol $q(x, \xi)$ satisfying $|\operatorname{Im} q(x, \xi)| \leq c \operatorname{Re} q(x, \xi)$ for some $c \geq 0$ and all $x, \xi \in \mathbb{R}^{d}$. Then, the condition in (3.1) is equivalent to

$$
\int_{B(0, r)} \frac{d \xi}{\left(\sup _{x \in \mathbb{R}^{d}} \operatorname{Re} q(x, \xi)\right)^{\kappa+1}}<\infty \quad \text { for some } r>0,
$$

and the condition in (3.2) is equivalent to

$$
\int_{B(0, r)} \frac{d \xi}{\left(\inf _{x \in \mathbb{R}^{d}}|q(x, \xi)|\right)^{\kappa+1}}<\infty \text { for some } r>0 .
$$

Proof. The assertion easily follows from the following inequalities

$$
\operatorname{Re} q(x, \xi) \leq \sqrt{(\operatorname{Re} q(x, \xi))^{2}+(\operatorname{Im} q(x, \xi))^{2}}=|q(x, \xi)| \leq \sqrt{1+c^{2}} \operatorname{Re} q(x, \xi), \quad x, \xi \in \mathbb{R}^{d} .
$$

In Proposition 2.7 we discussed conditions under which the relation in (2.3) (and hence in (3.1)) does not depend on $r>0$. By the same reasoning as before, we get that (3.2) does not depend on $r>0$ if

$$
\inf _{r_{0} \leq|\xi| \leq r} \inf _{x \in \mathbb{R}^{d}} \operatorname{Re} q(x, \xi)>0, \quad r>r_{0}>0 .
$$

Let us remark that the above condition is satisfied if

$$
\inf _{|\xi|=1} \inf _{x \in \mathbb{R}^{d}}\left(\langle\xi, C(x) \xi\rangle+\int_{B\left(0, \frac{1}{r}\right)}\langle\xi, y\rangle^{2} \nu(x, d y)\right)>0, \quad r>r_{0}>0,
$$

(see [San14a]). In addition, if we assume that the function $\xi \longmapsto \inf _{x \in \mathbb{R}^{d}} \sqrt{\operatorname{Re} q(x, \xi)}$ is radial and subadditive (that is, $\inf _{x \in \mathbb{R}^{d}} \sqrt{\operatorname{Re} q(x, \xi+\eta)} \leq \inf _{x \in \mathbb{R}^{d}} \sqrt{\operatorname{Re} q(x, \xi)}+\inf _{x \in \mathbb{R}^{d}} \sqrt{\operatorname{Re} q(x, \eta)}$ for all $\xi, \eta \in \mathbb{R}^{d}$ ), then the condition in (3.2) do not depend on $r>0$ (see [San14a, Proposition 2.4]).

In the following proposition we discuss the dependence of the $\kappa$-weak and $\kappa$-strong transience on the dimension of the state space.

Proposition 3.2. Let $\kappa>0$ and let $\left\{F_{t}\right\}_{t \geq 0}$ be a Lévy-type process with symbol $q(x, \xi)$.

(i) If

$$
\limsup _{|\xi| \longrightarrow 0} \frac{\sup _{x \in \mathbb{R}^{d}}|q(x, \xi)|}{|\xi|^{\gamma}}<\infty
$$

and $d \leq(\kappa+1) \gamma$ for some $\gamma>0$, then (3.1) holds true. 
(ii) If

$$
\liminf _{|\xi| \longrightarrow 0} \frac{\inf _{x \in \mathbb{R}^{d}} \operatorname{Re} q(x, \xi)}{|\xi|^{\gamma}}>0
$$

and $d>(\kappa+1) \gamma$ for some $\gamma>0$, then (3.2) holds true.

Proof. (i) By assumption, there exist constants $c>0$ (large enough) and $r>0$ (small enough), such that

$$
\int_{B(0, r)} \frac{d \xi}{\left(\sup _{x \in \mathbb{R}^{d}}|q(x, \xi)|\right)^{\kappa+1}} \geq \frac{1}{c} \int_{B(0, r)} \frac{d \xi}{|\xi|^{(\kappa+1) \gamma}}=\frac{S_{d}}{c} \int_{0}^{r} \rho^{d-1-(\kappa+1) \gamma} d \rho .
$$

Here, $S_{d}$ denotes the surface of a $d$-dimensional unit ball.

(ii) By assumption, there exist constants $c>0$ and $r>0$ (small enough), such that

$$
\int_{B(0, r)} \frac{d \xi}{\left(\inf _{x \in \mathbb{R}^{d}} \operatorname{Re} q(x, \xi)\right)^{\kappa+1}} \leq \frac{1}{c} \int_{B(0, r)} \frac{d \xi}{|\xi|^{(\kappa+1) \gamma}}=\frac{S_{d}}{c} \int_{0}^{r} \rho^{d-1-(\kappa+1) \gamma} d \rho .
$$

As a direct consequence of the above proposition we get the following.

Theorem 3.3. Let $\kappa>0$ and let $\left\{F_{t}\right\}_{t \geq 0}$ be a Lévy-type process with symbol $q(x, \xi)$ and Lévy triplet $(b(x), C(x), \nu(x, d y))$.

(i) If $q(x, \xi)=q(x,-\xi)$ for all $x, \xi \in \mathbb{R}^{d}$ (that is, $b(x)=0$ and $\nu(x, d y)$ is a symmetric measure for all $\left.x \in \mathbb{R}^{d}\right), d \leq 2(\kappa+1)$ and

$$
\sup _{x \in \mathbb{R}^{d}} \int_{\mathbb{R}^{d}}|y|^{2} \nu(x, d y)<\infty
$$

then (3.1) holds true.

(ii) If $d>2(\kappa+1)$ and

$$
\liminf _{|\xi| \longrightarrow 0} \frac{\inf _{x \in \mathbb{R}^{d}}\left(\langle\xi, C(x) \xi\rangle+\int_{\left\{|y| \leq \frac{\pi}{2|\xi|}\right\}}\langle\xi, y\rangle^{2} \nu(x, d y)\right)}{|\xi|^{2}}>0,
$$

then (3.2) holds true.

Proof. (i) First, observe that

$$
\begin{aligned}
\sup _{x \in \mathbb{R}^{d}}|q(x, \xi)| & =\sup _{x \in \mathbb{R}^{d}}\left(\langle\xi, C(x) \xi\rangle+\int_{\mathbb{R}^{d}}(1-\cos \langle\xi, y\rangle) \nu(x, d y)\right) \\
& \leq|\xi|^{2}\left(d \sup _{x \in \mathbb{R}^{d}} \max _{1 \leq i, j \leq d}\left|c_{i j}(x)\right|+\sup _{x \in \mathbb{R}^{d}} \int_{\mathbb{R}^{d}}|y|^{2} \nu(x, d y)\right),
\end{aligned}
$$

where we used the facts that for an arbitrary square matrix $M=\left(m_{i j}\right)_{1 \leq i, j \leq d}$ and $v \in \mathbb{R}^{d}$, $|\langle v, M v\rangle| \leq|v||M v| \leq d \max _{1 \leq i, j \leq d}\left|m_{i j}\right||v|^{2}$ and $1-\cos u \leq u^{2}$ for all $u \in \mathbb{R}$. Now, the claim follows as a direct consequence of Proposition 3.2. 
(ii) By employing the fact that $1-\cos u \geq u^{2} / \pi$ for all $|u| \leq \pi / 2$,

$$
\begin{aligned}
\inf _{x \in \mathbb{R}^{d}} \operatorname{Re} q(x, \xi) & =\inf _{x \in \mathbb{R}^{d}}\left(\langle\xi, c(x) \xi\rangle+\int_{\mathbb{R}^{d}}(1-\cos \langle\xi, y\rangle) \nu(x, d y)\right) \\
& \geq \frac{1}{\pi} \inf _{x \in \mathbb{R}^{d}}\left(\langle\xi, c(x) \xi\rangle+\int_{\left\{|y| \leq \frac{\pi}{2|\xi|}\right\}}\langle\xi, y\rangle^{2} \nu(x, d y)\right),
\end{aligned}
$$

which together with Proposition 3.2 proves the assertion.

Intuitively, the relation in (3.3) actually says that the underlying Lévy-type process in nondegenerate, that is, it either has a non-degenerate diffusion part or non-degenerate jump part. Now, we give some applications of the above results.

Example 3.4 (Elliptic diffusion processes). Assume that the functions $b=\left(b_{i}\right)_{1 \leq i \leq d}: \mathbb{R}^{d} \longrightarrow \mathbb{R}^{d}$ and $C=\left(c_{i j}\right)_{1 \leq i, j \leq d}: \mathbb{R}^{d} \longrightarrow \mathbb{R}^{d} \times \mathbb{R}^{d}$ satisfy the following:

(i) $b(x)$ is bounded and continuous;

(ii) $C(x)$ is bounded, symmetric and Lipschitz continuous;

(ii) for some constant $c>0$ and all $\xi \in \mathbb{R}^{d}$, $\inf _{x \in \mathbb{R}^{d}}\langle\xi, C(x) \xi\rangle \geq c|\xi|^{2}$.

Then, according to [RW00b, Theorem V.24.1] and [ST97, Theorem 2.3], there exists a unique open-set irreducible elliptic diffusion process satisfying conditions (C1), (C2) and (C3) (hence a Lévy-type process), with symbol of the form

$$
q(x, \xi)=-i\langle\xi, b(x)\rangle+\frac{1}{2}\langle\xi, C(x) \xi\rangle, \quad x, \xi \in \mathbb{R}^{d} .
$$

Fix $\kappa>0$. Then,

(i) if $\sup _{x \in \mathbb{R}^{d}}|b(x)|>0$ and $d \leq(\kappa+1)$, Proposition 3.2 implies that (3.1) holds true.

(ii) if $b(x)=0$ for all $x \in \mathbb{R}^{d}$ and $d \leq 2(\kappa+1)$, Theorem 3.3 entails that (3.1) holds true.

(iii) if $d>2(\kappa+1)$, Theorem 3.3 implies that (3.2) holds true.

Let us remark that if $d \leq 2$ and $b(x)=0$ for all $x \in \mathbb{R}^{d}$, then the underlying elliptic diffusion process satisfies the Chung-Fuch type condition for recurrence given in [San14a, Theorem 1.2] and if $d \geq 3$ it is always transient (see [San14a, Theorem 2.9]). In particular, a standard $d$-dimensional, $d \geq 3$, zero drift Brownian motion is $\kappa$-weakly transient if, and only if, $d \leq 2(\kappa+1)$.

Example 3.5 (Stable-like processes). Let $\alpha: \mathbb{R}^{d} \longrightarrow(0,2), \beta: \mathbb{R}^{d} \longrightarrow \mathbb{R}^{d}$ and $\gamma: \mathbb{R}^{d} \longrightarrow(0, \infty)$ be such that:

(i) $\alpha, \beta, \gamma \in C_{b}^{1}\left(\mathbb{R}^{d}\right)$;

(ii) $0<\underline{\alpha}:=\inf _{x \in \mathbb{R}^{d}} \alpha(x) \leq \sup _{x \in \mathbb{R}^{d}} \alpha(x)=: \bar{\alpha}<2$ and $\inf _{x \in \mathbb{R}^{d}} \gamma(x)>0$.

Here, $C_{b}^{k}\left(\mathbb{R}^{d}\right), k \geq 0$, denotes the space of $k$ times differentiable functions such that all derivatives up to order $k$ are bounded. Then, under this assumptions, in [Bas88], [Kol00, Theorem 5.1] and [SW13, Theorems 1.1 and 3.3] it has been shown that there exists a unique open-set irreducible Lévy-type process $\left\{F_{t}\right\}_{t \geq 0}$, called a stable-like process, determined by a symbol of the form $q(x, \xi)=$ $-i\langle\xi, \beta(x)\rangle+\gamma(x)|\xi|^{\alpha(x)}$ for $x, \xi \in \mathbb{R}^{d}$. Note that when $\alpha(x), \beta(x)$ and $\gamma(x)$ are constant functions, then we deal with a rotationally invariant stable Lévy process with drift. Again, fix $\kappa>0$. Then, Proposition 3.2 implies that 
(i) if $\sup _{x \in \mathbb{R}^{d}}|\beta(x)|>0, \underline{\alpha}<1$ and $d \leq(\kappa+1) \underline{\alpha},(3.1)$ holds true.

(ii) if $\sup _{x \in \mathbb{R}^{d}}|\beta(x)|>0, \underline{\alpha} \geq 1$ and $d \leq(\kappa+1)$, (3.1) holds true.

(iii) if $\beta(x)=0$ for all $x \in \mathbb{R}^{d}$ and $d \leq(\kappa+1) \underline{\alpha}$, (3.1) holds true.

(iv) if $d>(\kappa+1) \bar{\alpha},(3.2)$ holds true.

Let also remark that

(i) if $d=1, \beta(x)=0$ for all $x \in \mathbb{R}^{d}$ and $\underline{\alpha} \geq 1$, then $\left\{F_{t}\right\}_{t \geq 0}$ satisfies the Chung-Fuchs type condition for recurrence given in [San14a, Theorem 1.2].

(ii) if $d=1$ and $\bar{\alpha}<1$, then $\left\{F_{t}\right\}_{t \geq 0}$ is transient.

(iii) if $d \geq 2$, then $\left\{F_{t}\right\}_{t \geq 0}$ is always transient.

(see [San14a, Theorem 2.10]). In particular, a $d$-dimensional rotationally invariant $\alpha$-stable Lévy process is $\kappa$-weakly transient if, and only if, $d /(\kappa+1) \leq \alpha<d$.

The concept of the indices of stability can be generalized to general Lévy-type process through the so-called Pruitt indices (see [Pru81]). The Pruitt indices, for a Lévy-type process $\left\{F_{t}\right\}_{t \geq 0}$ with symbol $q(x, \xi)$, are defined in the following way

$$
\begin{aligned}
& \underline{\delta}:=\sup \left\{\delta \geq 0: \lim _{|\xi| \longrightarrow 0} \frac{\sup _{x \in \mathbb{R}^{d}}|q(x, \xi)|}{|\xi|^{\delta}}=0\right\} \\
& \bar{\delta}:=\sup \left\{\delta \geq 0: \lim _{|\xi| \longrightarrow 0} \frac{\inf _{x \in \mathbb{R}^{d}} \operatorname{Re} q(x, \xi)}{|\xi|^{\delta}}=0\right\}
\end{aligned}
$$

(see [Sch98b]). Note that $0 \leq \underline{\delta} \leq \bar{\delta}, \underline{\delta} \leq 2$ and in the case of a stable-like processes, we have $\underline{\delta}=\underline{\alpha}$ and $\bar{\delta}=\bar{\alpha}$. Now, we generalize Example 3.5 in terms of the Pruitt indices.

Theorem 3.6. Let $\kappa>0$ and let $\left\{F_{t}\right\}_{t \geq 0}$ be a Lévy-type process with symbol $q(x, \xi)$.

(i) If $d<(\kappa+1) \underline{\delta}$, then the condition in (3.1) holds true.

(ii) If $q(x, \xi)$ satisfies the condition in (3.2), then $d \geq(\kappa+1) \bar{\delta}$.

Proof. (i) Let $d /(\kappa+1) \leq \delta<\underline{\delta}$ be arbitrary. Then, by the definition of $\underline{\delta}$,

$$
\lim _{|\xi| \rightarrow 0} \frac{\sup _{x \in \mathbb{R}^{d}}|q(x, \xi)|}{|\xi|^{\delta}}=0 .
$$

Now, the claim easily follows by employing completely the same arguments as in the proof of Proposition 3.2.

(ii) Let us assume that this is not the case. Then, for all $d /(\kappa+1) \leq \delta<\bar{\delta}$, by the definition of $\bar{\delta}$, we have

$$
\lim _{|\xi| \longrightarrow 0} \frac{\inf _{x \in \mathbb{R}^{d}} \operatorname{Re} q(x, \xi)}{|\xi|^{\delta}}=0 .
$$

Consequently,

$$
\int_{B(0, r)} \frac{d \xi}{\left(\inf _{x \in \mathbb{R}^{d}} \operatorname{Re} q(x, \xi)\right)^{\kappa+1}} \geq \int_{B(0, r)} \frac{d \xi}{|\xi|^{(\kappa+1) \delta}}=\infty
$$

for all $r>0$ small enough. 
Let us remark that in general it is not possible to obtain the equivalence in Theorem 3.6. To see this, first recall that for two symmetric measures $\mu(d x)$ and $\bar{\mu}(d x)$ on $\mathcal{B}(\mathbb{R})$ which are finite outside of any neighborhood around the origin, we say that $\mu(d x)$ has a bigger tail than $\bar{\mu}(d x)$ if there exists $x_{0}>0$ such that $\mu(x, \infty) \geq \bar{\mu}(x, \infty)$ for all $x \geq x_{0}$. Now, fix $\kappa>0$ and let $\bar{\nu}(d x)$ be the Lévy measure of a one-dimensional symmetric $\alpha$-stable, $\alpha(\kappa+1)<1$, Lévy process $\left\{\bar{L}_{t}\right\}_{t \geq 0}$. Hence, $\left\{\bar{L}_{t}\right\}_{t \geq 0}$ is $\kappa$-strongly transient. Next, by [Sat99, Theorem 38.4], there exists a one-dimensional symmetric Lévy process $\left\{L_{t}\right\}_{t \geq 0}$ with Lévy measure $\nu(d x)$ having a bigger tail then $\bar{\nu}(d x)$ and satisfying the condition in (3.1). Moreover, $\left\{L_{t}\right\}_{t \geq 0}$ can be constructed such that it is recurrent. Consequently, by Fubini's theorem, for any $\delta>0$ we have

$$
\begin{aligned}
\int_{\left\{x>x_{0}\right\}} x^{\delta} \nu(d x) & =\int_{\left\{x>x_{0}\right\}} \int_{0}^{x} \delta y^{\delta-1} d y \nu(d x) \\
& =x_{0}^{\delta} \nu\left(x_{0}, \infty\right)+\delta \int_{\left\{y>x_{0}\right\}} y^{\delta-1} \nu(y, \infty) d y \\
& \geq x_{0}^{\delta} \nu\left(x_{0}, \infty\right)+\delta \int_{\left\{y>x_{0}\right\}} y^{\delta-1} \bar{\nu}(y, \infty) d y \\
& \geq x_{0}^{\delta} \nu\left(x_{0}, \infty\right)-x_{0}^{\delta} \bar{\nu}\left(x_{0}, \infty\right)+\int_{\left\{x>x_{0}\right\}} x^{\delta} \bar{\nu}(d x) .
\end{aligned}
$$

Hence, if $\int_{\{x>1\}} x^{\delta} \bar{\nu}(d y)=\infty$, then $\int_{\{x>1\}} x^{\delta} \nu(d y)=\infty$. Specially, according to [Sch98b, Prposition 5.4], $\underline{\delta}_{L} \leq \underline{\delta}_{\bar{L}}=\alpha$. To see that $d \geq \bar{\delta}(\kappa+1)$ does not automatically imply (3.2), we proceed in a similar way. Fix $\kappa>0$ and let $\bar{\nu}(d x)$ be the Lévy measure of a one-dimensional symmetric $\alpha$-stable, $\alpha(\kappa+1)<1$, Lévy process $\left\{\bar{L}_{t}\right\}_{t \geq 0}$. Again, by [Sat99, Theorem 38.4], there exists a one-dimensional symmetric Lévy process $\left\{L_{t}\right\}_{t \geq 0}$ with Lévy measure $\nu(d x)$ having a bigger tail then $\bar{\nu}(d x)$ and satisfying the condition in (3.1). But, by the same reasoning as above, we conclude that $\bar{\delta}_{L} \leq \bar{\delta}_{\bar{L}}=\alpha$. However, by assuming certain regularities (convexity and concavity) of the symbol, we get the converse statements in Theorem 3.6.

Theorem 3.7. Let $\kappa>0$ and let $\left\{F_{t}\right\}_{t \geq 0}$ be a Lévy-type process with symbol $q(x, \xi)$.

(i) If $\kappa+1 \geq d$ and the functions $\xi \longmapsto \sup _{x \in \mathbb{R}^{d}}|q(x, \xi)|$ and $|\xi| \longmapsto \sup _{x \in \mathbb{R}^{d}}|q(x, \xi)|$ are radial and convex, respectively, on some neighborhood of the origin, then $\underline{\delta}(\kappa+1) \geq d$.

(ii) If $\kappa+1 \leq d$ and the functions $\xi \longmapsto \sup _{x \in \mathbb{R}^{d}}|q(x, \xi)|$ and $|\xi| \longmapsto \sup _{x \in \mathbb{R}^{d}}|q(x, \xi)|$ are radial and concave, respectively, on some neighborhood of the origin, then $\underline{\delta}(\kappa+1) \leq d$. In addition, if the condition in (3.1) holds true, then $d=\kappa+1$.

(iii) If $\kappa+1 \geq d$ and the functions $\xi \longmapsto \inf _{x \in \mathbb{R}^{d}} \operatorname{Re} p(x, \xi)$ and $|\xi| \longmapsto \inf _{x \in \mathbb{R}^{d}} \operatorname{Re} p(x, \xi)$ are radial and convex, respectively, on some neighborhood of the origin, then $\bar{\delta}(\kappa+1) \geq d$. In addition, if the condition in (3.2) holds true, then $d=\kappa+1$.

(iv) If $\kappa+1 \leq d$ and the functions $\xi \longmapsto \inf _{x \in \mathbb{R}^{d}} \operatorname{Re} p(x, \xi)$ and $|\xi| \longmapsto \inf _{x \in \mathbb{R}^{d}} \operatorname{Re} p(x, \xi)$ are radial and concave, respectively, on some neighborhood of the origin, then $\bar{\delta}(\kappa+1) \leq d$. In addition, if $\kappa+1<d$, then the condition in (3.2) holds true. 
Proof. (i) Let $\varepsilon>0$ be such that $|\xi| \longmapsto \sup _{x \in \mathbb{R}^{d}}|q(x, \xi)|$ is convex on $[0, \varepsilon)$. Then, for all $\delta<1$,

$$
\begin{aligned}
\limsup _{|\xi| \longrightarrow 0} \frac{\sup _{x \in \mathbb{R}^{d}}|q(x, \xi)|}{|\xi|^{\delta}} & =\limsup _{|\xi| \longrightarrow 0} \frac{\sup _{x \in \mathbb{R}^{d}}\left|q\left(x, \frac{2|\xi|}{\varepsilon} \frac{\varepsilon \xi}{2|\xi|}\right)\right|}{|\xi|^{\delta}} \\
& \leq \limsup _{|\xi| \longrightarrow 0} \frac{2|\xi|^{1-\delta}}{\varepsilon} \sup _{x \in \mathbb{R}^{d}}\left|q\left(x, \frac{\varepsilon \xi}{2|\xi|}\right)\right|=0,
\end{aligned}
$$

where in the second step we used the convexity property. Thus, $\underline{\delta} \geq 1 \geq d /(\kappa+1)$.

(ii) Let $\varepsilon>0$ be such that $|\xi| \longmapsto \sup _{x \in \mathbb{R}^{d}}|q(x, \xi)|$ is concave on $[0, \varepsilon)$. Then, for all $\delta \geq 1$,

$$
\begin{aligned}
\liminf _{|\xi| \longrightarrow 0} \frac{\sup _{x \in \mathbb{R}^{d}} q(x, \xi)}{|\xi|^{\delta}} & =\liminf _{|\xi| \longrightarrow 0} \frac{\sup _{x \in \mathbb{R}^{d}}\left|q\left(x, \frac{2|\xi|}{\varepsilon} \frac{\varepsilon \xi}{2|\xi|}\right)\right|}{|\xi|^{\delta}} \\
& \geq \liminf _{|\xi| \longrightarrow 0} \frac{2|\xi|^{1-\delta}}{\varepsilon} \sup _{x \in \mathbb{R}^{d}}\left|q\left(x, \frac{\varepsilon \xi}{2|\xi|}\right)\right|>0,
\end{aligned}
$$

where in the second step we applied the concavity property. Hence, $\underline{\delta} \leq 1 \leq d /(1+\kappa)$. The second assertion easily follows from the above relation and the same arguments employed in the proof of Proposition 3.2.

(iii) The proof proceeds similarly as in (i).

(iv) The proof proceeds similarly as in (ii).

\section{Algebraic Weak and Strong Transience of Rotationally Invariant Lévy-Type Processes}

In this section, we discuss the $\kappa$-weak and $\kappa$-strong transience of a class of Lévy-type processes whose symbol is radial in the co-variable. In particular, if $(b(x), C(x), \nu(x, d y))$ denotes the corresponding Lévy triplet, [Sat99, Exercise 18.3] implies that this property is equivalent to

(i) $b(x)=0$ for all $x \in \mathbb{R}^{d}$;

(ii) $C(x)=c(x) I$ for some Borel measurable function $c: \mathbb{R}^{d} \longrightarrow[0, \infty)$, where $I$ is the $d \times d$ identity matrix;

(iii) $\nu(x, d y)=\nu(x, O d y)$ for all $x \in \mathbb{R}^{d}$ and all orthogonal $d \times d$ matrices $O$.

Specially, due to Proposition 2.7, these conditions entail that the condition in (3.1) does not depend on $r>0$. By following the proof of [San15, Theorem 3.2] we get the following "perturbation" result.

Theorem 4.1. Let $\kappa>0$ and let $\left\{F_{t}\right\}_{t \geq 0}$ and $\left\{\bar{F}_{t}\right\}_{t \geq 0}$ be d-dimensional Lévy-type processes with symbols $q(x, \xi)$ and $\bar{q}(x, \xi)$ and Lévy triplets $(0, c(x) I, \nu(x, d y))$ and $(0, \bar{c}(x) I, \bar{\nu}(x, d y))$, respectively. If there exists orthogonal matrix $O$ such that

$$
\sup _{x \in \mathbb{R}^{d}} \int_{\mathbb{R}^{d}}|y|^{2}|\nu(x, d y)-\bar{\nu}(O x, d y)|<\infty,
$$


then $q(x, \xi)$ satisfies (3.1) if, and only if, $\bar{q}(x, \xi)$ satisfies (3.1). Here, $|\mu(d y)|$ denotes the total variation measure of the signed measure $\mu(d y)$. If

$$
\liminf _{|\xi| \longrightarrow 0} \frac{\inf _{x \in \mathbb{R}^{d}} q(x, \xi)}{|\xi|^{2}}>\frac{1}{2} \sup _{x \in \mathbb{R}^{d}}|c(x)-\bar{c}(O x)|+\sup _{x \in \mathbb{R}^{d}} \int_{\mathbb{R}^{d}}|y|^{2}|\nu(x, d y)-\bar{\nu}(O x, d y)|,
$$

then, under (4.1), $q(x, \xi)$ satisfies (3.2) if, and only if, $q(x, \xi)$ satisfies (3.2).

Let us remark here that if $\nu(x, d y)$ is the Lévy measure of a Lévy-type process $\left\{F_{t}\right\}_{t \geq 0}$, then $\nu(O x, d y)=\nu(O x, O d y)$ is a Lévy measure of the Lévy-type process $\left\{O^{-1} F_{t}\right\}_{t \geq 0}$ (see [San 15 , Proposition 3.1]). Further, observe that in the Lévy process case the condition in (4.2) will be satisfied if, and only if, $\int_{\mathbb{R}^{d}}|y|^{2} \nu(d y)=\infty$. A situation where the condition in (4.1) trivially holds true is given in the following proposition.

Proposition 4.2. Let $\left\{F_{t}\right\}_{t \geq 0}$ and $\left\{\bar{F}_{t}\right\}_{t \geq 0}$ be d-dimensional Lev́y-type processes with Lévy measures $\nu(x, d y)$ and $\bar{\nu}(x, d y)$, respectively. If there exist an orthogonal matrix $O$ and $r>0$, such that $\nu(x, B)=\bar{\nu}(O x, B)$ for all $x \in \mathbb{R}^{d}$ and all $B \in \mathcal{B}\left(\mathbb{R}^{d}\right), B \subseteq B^{c}(0, r)$, then the condition in (4.1) holds true.

Note that Proposition 4.2 implies that the $\kappa$-weak and $\kappa$-strong transience of Lévy-type processes, satisfying the conditions from Theorem 4.1, depend only on big jumps, that is, they do not depend on the continuous part of the process and small jumps.

In the sequel, we derive some conditions for the $\kappa$-weak and $\kappa$-strong transience in terms of the Lévy measures. By a straightforward adaptation of [San15, Theorem 4.4], we conclude the following.

Theorem 4.3. Let $\kappa>0$ and let $\left\{F_{t}\right\}_{t \geq 0}$ be a Lévy-type process with Lévy measure $\nu(x, d y)$. Then,

$$
\int_{r}^{\infty} \frac{\rho^{2 \kappa-d+1}}{\left(\sup _{x \in \mathbb{R}^{d}} \int_{0}^{\rho} u \nu\left(x, B^{c}(0, u)\right) d u\right)^{\kappa+1}} d \rho=\infty \quad \text { for some (all) } r>0
$$

implies (3.1), and (3.2) implies

$$
\int_{r}^{\infty} \frac{\rho^{2 \kappa-d+1}}{\left(\inf _{x \in \mathbb{R}^{d}} \int_{0}^{\rho} u \nu\left(x, B^{c}(0, u)\right) d u\right)^{\kappa+1}} d \rho<\infty \quad \text { for some } r>0 .
$$

In addition, if the condition in (4.2) holds and there exists $u_{0} \geq 0$ such that $\nu(x, d y)=n(x,|y|) d y$ on $\mathcal{B}\left(B^{c}\left(0, u_{0}\right)\right)$ for some Borel function $n: \mathbb{R}^{d} \times(0, \infty) \longrightarrow(0, \infty)$ which is decreasing on $\left(u_{0}, \infty\right)$ for all $x \in \mathbb{R}^{d}$, then (3.1) holds true if, and only if, (4.3) holds true and (3.2) holds true if, and only if, (4.4) holds true.

Let us remark that in general we cannot conclude the equivalence in Theorem 4.3 (see the discussion after Theorem 3.6). By employing the integration by parts formula and Fatou's lemma, 
for any $x \in \mathbb{R}^{d}$, any $\rho>0$ and any $0<\varepsilon<\rho$, we have

$$
\begin{aligned}
& \frac{\rho^{2}}{2} \nu\left(x, B^{c}(0, \rho)\right)+\frac{1}{2} \int_{B(0, \rho)}|y|^{2} \nu(x, d y) \\
& \geq \liminf _{\epsilon \longrightarrow 0}\left(\frac{\rho^{2}}{2} \nu\left(x, B^{c}(0, \rho)\right)-\frac{\epsilon^{2}}{2} \nu\left(x, B^{c}(0, \epsilon)\right)+\frac{1}{2} \int_{B(0, \rho) \cap B^{c}(0, \epsilon)}|y|^{2} \nu(x, d y)\right) \\
& =\liminf _{\epsilon \longrightarrow 0} \int_{\epsilon}^{\rho} u \nu\left(x, B^{c}(0, u)\right) d u \\
& \geq \int_{0}^{\rho} u \nu\left(x, B^{c}(0, u)\right) d u \\
& =\int_{0}^{\varepsilon} u \nu\left(x, B^{c}(0, u)\right) d u+\int_{\varepsilon}^{\rho} u \nu\left(x, B^{c}(0, u)\right) d u \\
& \geq \frac{\varepsilon^{2}}{2} \nu\left(x, B^{c}(0, \varepsilon)\right)+\frac{\rho^{2}}{2} \nu\left(x, B^{c}(0, \rho)\right)-\frac{\varepsilon^{2}}{2} \nu\left(x, B^{c}(0, \varepsilon)\right)+\frac{1}{2} \int_{B(0, \rho) \cap B^{c}(0, \varepsilon)}|y|^{2} \nu(x, d y) \\
& =\frac{\rho^{2}}{2} \nu\left(x, B^{c}(0, \rho)\right)+\frac{1}{2} \int_{B(0, \rho) \cap B^{c}(0, \varepsilon)}|y|^{2} \nu(x, d y) .
\end{aligned}
$$

Now, by letting $\varepsilon \longrightarrow 0$, Fatou's lemma yields

$$
\int_{0}^{\rho} u \nu\left(x, B_{u}^{c}(0)\right) d u=\frac{\rho^{2}}{2} \nu\left(x, B_{\rho}^{c}(0)\right)+\frac{1}{2} \int_{B_{\rho}(0)}|y|^{2} \nu(x, d y) .
$$

Thus, we have proved the following.

Proposition 4.4. Let $\kappa>0$ and let $\left\{F_{t}\right\}_{t \geq 0}$ be a Lévy-type process with Lévy measure $\nu(x, d y)$. Then, (4.3) holds if

$$
\int_{r}^{\infty} \frac{\rho^{2 \kappa-d+1}}{\left(\rho^{2} \sup _{x \in \mathbb{R}^{d}} \nu\left(x, B^{c}(0, \rho)\right)+\sup _{x \in \mathbb{R}^{d}} \int_{B(0, \rho)}|y|^{2} \nu(x, d y)\right)^{\kappa+1}} d \rho=\infty \quad \text { for some } r>0,
$$

and (4.4) holds if

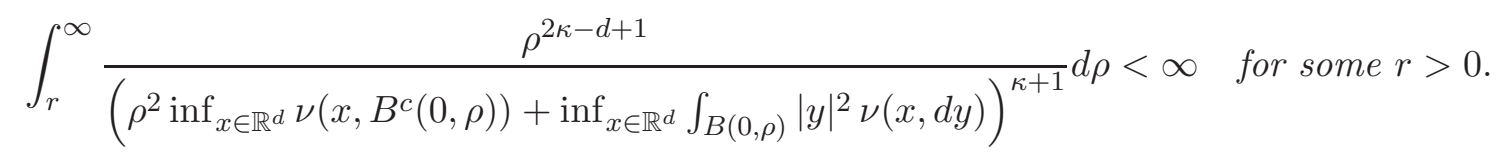

In particular, (4.4) holds if either one of the following conditions holds

$$
\int_{r}^{\infty} \frac{\rho^{-d-1}}{\left(\sup _{x \in \mathbb{R}^{d}} \nu\left(x, B^{c}(0, \rho)\right)\right)^{\kappa+1}} d \rho<\infty \quad \text { for some } r>0
$$

or

$$
\int_{r}^{\infty} \frac{\rho^{2 \kappa-d+1}}{\left(\inf _{x \in \mathbb{R}^{d}} \int_{B(0, \rho)}|y|^{2} \nu(x, d y)\right)^{\kappa+1}} d \rho<\infty \quad \text { for some } r>0 .
$$

As a direct consequence of the above proposition we get the following. 
Corollary 4.5. Let $\kappa>0$ and let $\left\{F_{t}\right\}_{t \geq 0}$ be a Lévy-type process with Lévy measure $\nu(x, d y)$. Assume that there exist $u_{0} \geq 0$ such that $\nu(x, d y)=n(x,|y|) d y$ on $\mathcal{B}\left(B^{c}\left(0, u_{0}\right)\right)$ for some Borel function $n: \mathbb{R}^{d} \times(0, \infty) \longrightarrow(0, \infty)$ which is decreasing on $\left(u_{0}, \infty\right)$ for all $x \in \mathbb{R}^{d}$. Then,

$$
\int_{r}^{\infty} \frac{\rho^{-d \kappa-2 d-1} d \rho}{\left(\inf _{x \in \mathbb{R}^{d}} n(x, \rho)\right)^{\kappa+1}}<\infty
$$

implies (4.4).

Proof. For any $r>u_{0}$, we have

$$
\begin{aligned}
\int_{r}^{\infty} \frac{\rho^{2 \kappa-d+1} d \rho}{\left(\inf _{x \in \mathbb{R}^{d}} \int_{B(0, \rho)}|y|^{2} \nu(x, d y)\right)^{\kappa+1}} & \leq \frac{1}{S_{d}^{\kappa+1}} \int_{r}^{\infty} \frac{\rho^{2 \kappa-d+1} d \rho}{\left(\inf _{x \in \mathbb{R}^{d}} \int_{u_{0}}^{\rho} u^{d+1} n(x, u) d u\right)^{\kappa+1}} \\
& \leq \frac{(d+2)^{\kappa+1}}{S_{d}^{\kappa+1}} \int_{r}^{\infty} \frac{\rho^{2 \kappa-d+1} d \rho}{\left(\rho^{d+2}-u_{0}^{d+2}\right)^{\kappa+1}\left(\inf _{x \in \mathbb{R}^{d}} n(x, \rho)\right)^{\kappa+1}} \\
& \leq c \int_{r}^{\infty} \frac{\rho^{-d \kappa-2 d-1} d \rho}{\left(\inf _{x \in \mathbb{R}^{d}} n(x, \rho)\right)^{\kappa+1}},
\end{aligned}
$$

where in the second step we employed the fact that $n(x, u)$ is decreasing in $u$ on $\left(u_{0}, \infty\right)$ and $c>(d+2)^{\kappa+1} r^{(d+2)(\kappa+1)} / S_{d}^{\kappa+1}\left(r^{d+2}-u_{0}^{d+2}\right)^{\kappa+1}$ is arbitrary. Now, the claim is a direct consequence of Proposition 4.4.

In the following proposition we slightly generalize Theorem 4.3.

Proposition 4.6. Let $\kappa>0$ and let $\left\{F_{t}\right\}_{t \geq 0}$ be a Lévy-type process with symbol $q(x, \xi)$ and Lévy triplet $(0, c(x) I, \nu(x, d y))$, satisfying

$$
\liminf _{|\xi| \longrightarrow 0} \inf _{x \in \mathbb{R}^{d}} \int_{\mathbb{R}^{d}} \frac{1-\cos \langle\xi, y\rangle}{|\xi|^{2}} \nu(x, d y)>0 .
$$

Then, (4.8) implies (3.2).

Proof. First, because of the radiality of the function $\xi \longmapsto q(x, \xi)$ for all $x \in \mathbb{R}^{d}$,

$$
\begin{aligned}
q(x, \xi)-\frac{1}{2} c(x)|\xi|^{2} & =q\left(x,|\xi| e_{i}\right)-\frac{1}{2} c(x)|\xi|^{2} \\
& =\int_{\mathbb{R}^{d}}\left(1-\cos |\xi| y_{i}\right) \nu(x, d y) \\
& \geq \frac{1}{\pi}|\xi|^{2} \int_{B\left(0, \frac{1}{|\xi|}\right)} y_{i}^{2} \nu(x, d y) \\
& =\frac{1}{d \pi}|\xi|^{2} \int_{B\left(0, \frac{1}{|\xi|}\right)}|y|^{2} \nu(x, d y), \quad \xi \in \mathbb{R}^{d},
\end{aligned}
$$


where in the third step we used the fact that $1-\cos u \geq u^{2} / \pi$ for all $|u| \leq 1$. Now, for $r>0$,

$$
\begin{aligned}
\int_{B(0, r)} \frac{d \xi}{\left.\inf _{x \in \mathbb{R}^{d}}\left(q(x, \xi)-\frac{1}{2} c(x)|\xi|^{2}\right)\right)^{\kappa+1}} & \leq d^{\kappa+1} \pi^{\kappa+1} \int_{B(0, r)} \frac{d \xi}{\left(|\xi|^{2} \inf _{x \in \mathbb{R}^{d}} \int_{B\left(0, \frac{1}{|\xi|}\right)}|y|^{2} \nu(x, d y)\right)^{\kappa+1}} \\
& =S_{d} d^{\kappa+1} \pi^{\kappa+1} \int_{0}^{r} \frac{\rho^{d-1} d \rho}{\left(\rho^{2} \inf _{x \in \mathbb{R}^{d}} \int_{B\left(0, \frac{1}{\rho}\right)}|y|^{2} \nu(x, d y)\right)^{\kappa+1}} \\
& =S_{d} d^{\kappa+1} \pi^{\kappa+1} \int_{\frac{1}{r}}^{\infty} \frac{\rho^{2 \kappa-d+1} d \rho}{\left(\inf _{\left.x \in \mathbb{R}^{d} \int_{B(0, \rho)}|y|^{2} \nu(x, d y)\right)^{\kappa+1}} .\right.}
\end{aligned}
$$

Finally,

$$
\begin{aligned}
1 & \leq \liminf _{|\xi| \longrightarrow 0} \frac{\inf _{x \in \mathbb{R}^{d}} q(x, \xi)}{\inf _{x \in \mathbb{R}^{d}}\left(q(x, \xi)-\frac{1}{2} c(x)|\xi|^{2}\right)} \\
& \leq \limsup _{|\xi| \longrightarrow 0} \frac{\inf _{x \in \mathbb{R}^{d}} q(x, \xi)}{\inf _{x \in \mathbb{R}^{d}}\left(q(x, \xi)-\frac{1}{2} c(x)|\xi|^{2}\right)} \\
& \leq 1+\frac{\sup _{x \in \mathbb{R}^{d}} c(x)}{\liminf } \inf _{|\xi| \longrightarrow 0} \inf _{x \in \mathbb{R}^{d}} \int_{\mathbb{R}^{d}} \frac{1-\cos \langle\xi, y\rangle}{|\xi|^{2}} \nu(x, d y)
\end{aligned}
$$

which together with (4.10) completes the proof.

Let us now give some applications of the results presented above.

Example 4.7. Let $\left\{F_{t}\right\}_{t>0}$ be a $d$-dimensional stable-like process with symbol $q(x, \xi)=\gamma(x)|\xi|^{\alpha(x)}$. The Lévy measure of $\left\{F_{t}\right\}_{t \geq 0}$ is given by $\nu(x, d y)=\delta(x)|y|^{-d-\alpha(x)} d y$, where

$$
\delta(x):=\gamma(x) \frac{\alpha(x) 2^{\alpha(x)-1} \Gamma((\alpha(x)+1) / 2)}{\pi^{1 / 2} \Gamma(1-\alpha(x) / 2)}, \quad x \in \mathbb{R}^{d},
$$

and $\Gamma(x)$ denotes the Gamma function. Obviously, $\nu(x, d y)$ satisfies all the assumptions from Theorem 4.3. Thus, by a straightforward application of (4.3) and (4.4) we conclude the same conditions for the $\kappa$-weak and $\kappa$-strong transience of $\left\{F_{t}\right\}_{t \geq 0}$ obtained in Example 3.5.

Example 4.8. Let $\alpha, \gamma: \mathbb{R}^{d} \longrightarrow(0, \infty)$ be arbitrary bounded and continuous functions such that $\inf _{x \in \mathbb{R}^{d}} \alpha(x)>0$ and $\inf _{x \in \mathbb{R}^{d}} \gamma(x)>0$. Define $n: \mathbb{R}^{d} \times(0, \infty) \longrightarrow(0, \infty)$ by

$$
n(x, u):=\frac{\gamma(x)}{u^{d+\alpha(x)}} 1_{\{v: v \geq 1\}}(u) .
$$

Because of continuity of the functions $\alpha(x)$ and $\gamma(x)$, without loss of generality, we can assume that $\int_{\mathbb{R}^{d}} n(x,|y|) d y=1$ for all $x \in \mathbb{R}^{d}$. Now, there exists an open-set irreducible Lévy-type process determined by a Lévy measure and symbol of the form $\nu(x, d y):=n(x,|y|) d y$ and $q(x, \xi):=$ $\int_{\mathbb{R}^{d}}(1-\cos \langle\xi, y\rangle) \nu(x, d y)$, respectively. Indeed, let $\left\{F_{n}\right\}_{n \geq 0}$ be a $d$-dimensional Markov chain determined by a transition function of the form $p(x, d y):=n(x,|y-x|)$ and let $\left\{N_{t}\right\}_{t \geq 0}$ be a Poisson process (with parameter 1) independent of the chain $\left\{F_{n}\right\}_{n \geq 0}$. Then, clearly, the process $F_{t}:=F_{N_{t}}, t \geq 0$, is a strong Markov process with semigroup given by

$$
P_{t} f(x)=e^{-t} \sum_{n=0}^{\infty} \frac{t^{n}}{n !} \int_{\mathbb{R}^{d}} f(y) p^{n}(x, d y), \quad f \in B_{b}\left(\mathbb{R}^{d}\right) .
$$


First, observe that, due to the continuity of the functions $\alpha(x)$ and $\gamma(x)$ and [MT09, Proposition 6.1.1] (which states that $x \longmapsto \int_{\mathbb{R}^{d}} f(y) p(x, d y)$ is a continuous and bounded function for every $\left.f \in C_{b}\left(\mathbb{R}^{d}\right)\right), P_{t}\left(C_{b}\left(\mathbb{R}^{d}\right)\right) \subseteq C_{b}\left(\mathbb{R}^{d}\right), t \geq 0$. Here, $C_{b}\left(\mathbb{R}^{d}\right)$ denotes the space of continuous and bounded functions. Next, to see that $P_{t}\left(C_{\infty}\left(\mathbb{R}^{d}\right)\right) \subseteq C_{\infty}\left(\mathbb{R}^{d}\right), t \geq 0$, we proceed as follows. Fix $f \in C_{\infty}\left(\mathbb{R}^{d}\right)$ and $\varepsilon>0$. Since $C_{c}\left(\mathbb{R}^{d}\right)$ is dense in $\left(C_{\infty}\left(\mathbb{R}^{d}\right),\|\cdot\|_{\infty}\right)$, there exists $f_{\varepsilon} \in C_{c}\left(\mathbb{R}^{d}\right)$ such that $\left\|f-f_{\varepsilon}\right\|_{\infty}<\varepsilon$. Now, we have

$$
\begin{aligned}
\left|\int_{\mathbb{R}^{d}} f(y) p(x, d y)\right| & \leq \int_{\mathbb{R}^{d}}\left|f(y)-f_{\varepsilon}(y)+f_{\varepsilon}(y)\right| p(x, d y) \\
& \leq \int_{\mathbb{R}^{d}}\left|f_{\varepsilon}(y)\right| p(x, d y)+\varepsilon \\
& \leq\left\|f_{\varepsilon}\right\|_{\infty} \int_{\operatorname{supp} f_{\varepsilon}-x} n(x,|y|) d y+\varepsilon .
\end{aligned}
$$

Thus, due to the boundedness (away from zero and infinity) of the functions $\alpha(x)$ and $\gamma(x)$ and the fact that $\operatorname{supp} f_{\epsilon}$ is a compact set, we have that the function $x \longmapsto \int_{\mathbb{R}^{d}} f(y) p(x, d y)$ vanishes at infinity. In particular, $\left\{P_{t}\right\}_{t \geq 0}$ enjoys the Feller property. Finally, obviously $\left\{P_{t}\right\}_{t \geq 0}$ is also strongly continuous, the domain of the corresponding generator contains $C_{\infty}\left(\mathbb{R}^{d}\right)$ and the corresponding Lévy measure and symbol are given by $\nu(x, d y)$ and $q(x, \xi)$, respectively. To check open-set irreducibility of $\left\{F_{t}\right\}_{t \geq 0}$ is straightforward.

Put $\underline{\alpha}:=\inf _{x \in \mathbb{R}^{d}} \alpha(x) \leq \sup _{x \in \mathbb{R}^{d}} \alpha(x)=: \bar{\alpha}$. Recall that, due to [San14a, Theorems 2.8 and 3.9] and [San15, Theorem 4.4],

(i) if $d \leq 2$, then $\left\{F_{t}\right\}_{t \geq 0}$ is transient if $\bar{\alpha}<d$.

(ii) if $d \geq 3$, then $\left\{F_{t}\right\}_{t \geq 0}$ is always transient.

Next, fix $\kappa>0$. Then, by a straightforward application of Theorem 4.3, we have

(i) if $\bar{\alpha}<2$, then $\left\{F_{t}\right\}_{t \geq 0}$ satisfies (3.1) if $\underline{\alpha}(\kappa+1) \geq d$, and it satisfies (3.2) if $\bar{\alpha}(\kappa+1)<d$.

(ii) if $\bar{\alpha}=\underline{\alpha}=2$, then $\left\{F_{t}\right\}_{t \geq 0}$ satisfies (3.1) if $2(\kappa+1)>d$, and it satisfies $(3.2)$ if $2(\kappa+1) \leq d$.

(iii) if $\underline{\alpha}>2$, then $\left\{F_{t}\right\}_{t \geq 0}$ satisfies (3.1) if $2(\kappa+1) \geq d$, and it satisfies $(3.2)$ if $2(\kappa+1)<d$.

Example 4.9. Let $\left\{L_{t}\right\}_{t>0}$ be a $d$-dimensional Lévy process with Lévy measure of the form $\nu(d y)=$ $n(|y|) d y$, where $n:(0, \infty) \longrightarrow(0, \infty)$ is a decreasing (on $\left(u_{0}, \infty\right)$ for some $\left.u_{0} \geq 0\right)$ and regularly varying function with index $\delta \leq-d$ (that is, $\lim _{u \longrightarrow \infty} n(\lambda u) / n(u)=\lambda^{\delta}$ for all $\lambda>0$ ). First, recall that, according to [San14a, Theorems 3.9 and 3.11] and [San15, Proposition 4.5], $\left\{L_{t}\right\}_{t \geq 0}$ is transient if

(i) $d=1,2$, and $-2 d<\delta \leq-d$ or $\delta=-2 d$ and

$$
\int_{r} \frac{d \rho}{\rho^{2 d+1} n(\rho)}<\infty \quad \text { for some } r>0
$$

(ii) $d \geq 3$.

Further, observe that, due to [BGT87, Theorem 1.5.11], for any $-d-2 \leq \delta \leq-d$,

$$
\lim _{\rho \longrightarrow \infty} \frac{\nu\left(B_{\rho}^{c}(0)\right)}{\rho^{d} n(\rho)}=\frac{1}{S_{d}(-d-\delta)} \quad \text { and } \quad \lim _{\rho \longrightarrow \infty} \frac{\int_{B_{\rho}(0)}|y|^{2} \nu(d y)}{\rho^{d+2} n(\rho)}=\frac{1}{S_{d}(d+\delta+2)} .
$$

Consequently, Proposition 4.4, Corollary 4.5 and [BGT87, Proposition 1.3.6] yield that 
(i) if $d \geq 3$ and $\delta<-d-2$, then $\left\{L_{t}\right\}_{t \geq 0}$ is $\kappa$-weakly transient if, and only if, $2(\kappa+1) \geq d$.

(ii) if $d=1,2, \delta=-2 d$ and (4.11) holds, then $\left\{L_{t}\right\}_{t \geq 0}$ is $\kappa$-weakly transient if, and only if, $2(\kappa+1)>d$.

(iii) if $d \geq 3$ and $\delta=-d-2$, then $\left\{L_{t}\right\}_{t \geq 0}$ is $\kappa$-weakly transient if, and only if, $2(\kappa+1)>d$.

(iv) if $d=1$ and $-2<\delta<-1$, then $\left\{L_{t}\right\}_{t \geq 0}$ is $\kappa$-weakly transient if, and only if, $\kappa+2+\delta(\kappa+1) \leq 0$.

(v) if $d \geq 2$ and $-d-2<\delta<-d$, then $\left\{L_{t}\right\}_{t \geq 0}$ is $\kappa$-weakly transient if, and only if, $d(\kappa+2)+$ $\delta(\kappa+1) \leq 0$.

(vi) if $\delta=-d$, then $\left\{L_{t}\right\}_{t \geq 0}$ is always $\kappa$-strongly transient.

Finally, as a consequence of Theorem 4.3 we can conclude the following comparison conditions for the $\kappa$-weak and $\kappa$-strong transience.

Theorem 4.10. Let $\kappa>0$ and let $\left\{F_{t}\right\}_{t \geq 0}$ and $\left\{\bar{F}_{t}\right\}_{t \geq 0}$ be Lévy-type processes with symbols $q(x, \xi)$ and $\bar{q}(x, \xi)$ and Lévy measures $\nu(x, d y)$ and $\bar{\nu}(x, d y)$, respectively. Assume that there exists $u_{0} \geq 0$ such that:

(i) $\nu(x, d y)=n(x,|y|) d y$ on $\mathcal{B}\left(B^{c}\left(0, u_{0}\right)\right)$ for some Borel function $n: \mathbb{R}^{d} \times(0, \infty) \longrightarrow(0, \infty)$ which is decreasing on $\left(u_{0}, \infty\right)$ for all $x \in \mathbb{R}^{d}$;

(ii) $\nu\left(x, B^{c}(0, u)\right) \geq \bar{\nu}\left(x, B^{c}(0, u)\right)$ for all $x \in \mathbb{R}^{d}$ and all $u>u_{0}$.

Then,

$$
\int_{B(0, r)} \frac{d \xi}{\left(\sup _{x \in \mathbb{R}^{d}} q(x, \xi)\right)^{\kappa+1}}=\infty \quad \text { for all } r>0
$$

implies

$$
\int_{B(0, r)} \frac{d \xi}{\left(\sup _{x \in \mathbb{R}^{d}} \bar{q}(x, \xi)\right)^{\kappa+1}}=\infty \quad \text { for all } r>0 .
$$

In addition, if $q(x, \xi)$ satisfies $(4.2)$, then

$$
\int_{B(0, r)} \frac{d \xi}{\left(\inf _{x \in \mathbb{R}^{d}} \bar{q}(x, \xi)\right)^{\kappa+1}}<\infty \text { for some } r>0
$$

implies

$$
\int_{B(0, r)} \frac{d \xi}{\left(\inf _{x \in \mathbb{R}^{d}} q(x, \xi)\right)^{\kappa+1}}<\infty \quad \text { for some } r>0
$$

\section{Acknowledgement}

This work was supported by the Croatian Science Foundation under Grant 3526 and NEWFELPRO Programme under Grant 31. The author thanks the referee for helpful comments and careful corrections. 


\section{References}

[Bas88] R. F. Bass. Uniqueness in law for pure jump Markov processes. Probab. Theory Related Fields, 79(2):271-287, 1988.

[BC86] R. F. Bass and M. Cranston. The Malliavin calculus for pure jump processes and applications to local time. Ann. Probab., 14(2):490-532, 1986.

[BGT87] N. H. Bingham, C. M. Goldie, and J. L. Teugels. Regular variation. Cambridge University Press, Cambridge, 1987.

[BSW13] B. Böttcher, R. L. Schilling, and J. Wang. Lévy matters. III. Springer, Cham, 2013.

[Cou66] P. Courrége. Sur la forme intégro-différentielle des opérateus de $C_{K}^{\infty}$ dans $C$ satisfaisant au principe du maximum. Sém. Théorie du Potentiel, exposé 2:38 pp., 1965-1966.

[DGW05] D. A. Dawson, L. G. Gorostiza, and A. Wakolbinger. Degrees of transience and recurrence and hierarchical random walks. Potential Anal., 22(4):305-350, 2005.

[EK86] S. N. Ethier and T. G. Kurtz. Markov processes. John Wiley \& Sons Inc., New York, 1986.

[Haw77] J. Hawkes. Moments of last exit times. Mathematika, 24(2):266-269, 1977.

[Ish01] Y. Ishikawa. Density estimate in small time for jump processes with singular Lévy measures. Tohoku Math. J. (2), 53(2):183-202, 2001.

[Jac98] N. Jacob. Characteristic functions and symbols in the theory of Feller processes. Potential Anal., 8(1):61-68, 1998.

[Jac01] N. Jacob. Pseudo differential operators and Markov processes. Vol. I. Imperial College Press, London, 2001.

[Jac05] N. Jacob. Pseudo differential operators and Markov processes. Vol. III. Imperial College Press, London, 2005.

[KC99] Y. Kwon and Lee C. Strong Feller property and irreducibility of diffusions with jumps. Stochastics, 67(1-2):147-157, 1999.

[KM14] V. P. Knopova and Kulik A. M. The parametrix method and the weak solution to an SDE driven by an $\alpha$-stable noise. Preprint. Available at arXiv: 1412.1732, 2014.

[Kol00] V. N. Kolokoltsov. Symmetric stable laws and stable-like jump-diffusions. Proc. Lond. Math. Soc. (3), 80(3):725-768, 2000.

[KS12] V. P. Knopova and R. L. Schilling. Transition density estimates for a class of Lévy and Lévy-type processes. J. Theoret. Probab., 25(1):144-170, 2012.

[KS13] V. P. Knopova and R. L. Schilling. A note on the existence of transition probability densities of Lévy processes. Forum Math., 25(1):125-149, 2013.

[Kul07] A. M. Kulik. Stochastic calculus of variations for general Lévy processes and its applications to jump-type SDEs with non-degenerated drift. Preprint. Available at arXiv: $0606427,2007$. 
[LL06] B. Li and L. Liu. An estimate on the distribution and moments of the last exit time of an elliptic diffusion process. Acta Math. Sci. Ser. B Engl. Ed., 26(4):639-645, 2006.

[Mas07] H. Masuda. Ergodicity and exponential $\beta$-mixing bounds for multidimensional diffusions with jumps. Stochastic Process. Appl., 117(1):35-56, 2007.

[Mas09] H. Masuda. Erratum to: "Ergodicity and exponential $\beta$-mixing bound for multidimensional diffusions with jumps" [Stochastic Process. Appl. 117 (2007) 35-56]. Stochastic Process. Appl., 119(2):676-678, 2009.

[MT93] S. P. Meyn and R. L. Tweedie. Stability of Markovian processes. II. Continuous-time processes and sampled chains. Adv. in Appl. Probab., 25(3):487-517, 1993.

[MT09] S. Meyn and R. L. Tweedie. Markov chains and stochastic stability. Cambridge University Press, Cambridge, second edition, 2009.

[Pic96] J. Picard. On the existence of smooth densities for jump processes. Probab. Theory Related Fields, 105(4):481-511, 1996.

[Pic10] J. Picard. Erratum to: On the existence of smooth densities for jump processes. Probab. Theory Related Fields, 147(3-4):711-713, 2010.

[Por90] S. C. Port. Asymptotic expansions for the expected volume of a stable sausage. Ann. Probab., 18(2):492-523, 1990.

[Pru81] W. E. Pruitt. The growth of random walks and Lévy processes. Ann. Probab., 9(6):948956, 1981.

[RW00a] L. C. G. Rogers and D. Williams. Diffusions, Markov processes, and martingales. Vol. 1. Cambridge University Press, Cambridge, 2000.

[RW00b] L. C. G. Rogers and D. Williams. Diffusions, Markov processes, and martingales. Vol. 2. Cambridge Mathematical Library. Cambridge University Press, Cambridge, 2000.

[San13] N. Sandrić. Recurrence and transience property for a class of Markov chains. Bernoulli, 19(5B):2167-2199, 2013.

[San14a] N. Sandrić. Long-time behavior for a class of Feller processes. To appear in: Trans. Am. Math. Soc. Available at arXiv: 1401.2646, 2014.

[San14b] N. Sandrić. Recurrence and transience criteria for two cases of stable-like Markov chains. J. Theoret. Probab., 27(3):754-788, 2014.

[San15] N. Sandrić. On recurrence and transience of two-dimensional Lévy and Lévy-type processes. Preprint, 2015.

[Sat99] K. Sato. Lévy processes and infinitely divisible distributions, volume 68. Cambridge University Press, Cambridge, 1999.

[Sch98a] R. L. Schilling. Conservativeness and extensions of Feller semigroups. Positivity, 2:239$256,1998$.

[Sch98b] R. L. Schilling. Growth and Hölder conditions for the sample paths of Feller processes. Probab. Theory Related Fields, 112(4):565-611, 1998. 
[She91] S.-J. Sheu. Some estimates of the transition density of a nondegenerate diffusion Markov process. Ann. Probab., 19(2):538-561, 1991.

[ST97] O. Stramer and R. L. Tweedie. Existence and stability of weak solutions to stochastic differential equations with non-smooth coefficients. Statist. Sinica, 7(3):577-593, 1997.

[SW04] K. Sato and T. Watanabe. Moments of last exit times for Lévy processes. Ann. Inst. H. Poincaré Probab. Statist., 40(2):207-225, 2004.

[SW05] K. Sato and T. Watanabe. Last exit times for transient semistable processes. Ann. Inst. H. Poincaré Probab. Statist., 41(5):929-951, 2005.

[SW12] R. L. Schilling and J. Wang. Strong Feller continuity of Feller processes and semigroups. Infin. Dimens. Anal. Quantum Probab. Relat. Top., 15(2):1250010, 28, 2012.

[SW13] R. L. Schilling and J. Wang. Some theorems on Feller processes: transience, local times and ultracontractivity. Trans. Amer. Math. Soc., 365(6):3255-3268, 2013.

[SY84] K. Sato and M. Yamazato. Operator-selfdecomposable distributions as limit distributions of processes of Ornstein-Uhlenbeck type. Stochastic Process. Appl., 17(1):73-100, 1984.

[Tak67] J. Takeuchi. Moments of the last exit times. Proc. Japan Acad., 43:355-360, 1967.

[TT79] P. Tuominen and R. L. Tweedie. The recurrence structure of general Markov processes. Proc. London Math. Soc. (3), 39(3):554-576, 1979.

[Twe94] R. L. Tweedie. Topological conditions enabling use of Harris methods in discrete and continuous time. Acta Appl. Math., 34(1-2):175-188, 1994.

[WZL06] C. Wu, F. Zhang, and L. Liu. Criteria of strong transience for operator-self-similar Markov processes. Acta Math. Sci. Ser. B Engl. Ed., 26(1):41-48, 2006.

[Yam98] K. Yamamuro. On transient Markov processes of Ornstein-Uhlenbeck type. Nagoya Math. J., 149:19-32, 1998. 